\title{
ON THE LOWER SEMICONTINUOUS QUASICONVEX ENVELOPE FOR UNBOUNDED INTEGRANDS (I)
}

\author{
Marcus WAGNER ${ }^{1}$
}

\begin{abstract}
Motivated by the study of multidimensional control problems of Dieudonné-Rashevsky type, we raise the question how to understand to notion of quasiconvexity for a continuous function $f$ with a convex body $\mathrm{K} \subset \mathbb{R}^{n m}$ instead of the whole space $\mathbb{R}^{n m}$ as the range of definition. In the present paper, we trace the consequences of an infinite extension of $f$ outside $\mathrm{K}$, and thus study quasiconvex functions which are allowed to take the value $+\infty$. As an appropriate envelope, we introduce and investigate the lower semicontinuous quasiconvex envelope

$$
f^{(q c)}(v)=\sup \left\{g(v) \mid g: \mathbb{R}^{n m} \rightarrow \mathbb{R} \cup\{+\infty\}\right. \text { quasiconvex and lower semicontinuous, }
$$$$
\left.g(v) \leq f(v) \quad \forall v \in \mathbb{R}^{n m}\right\} .
$$

Our main result is a representation theorem for $f^{(q c)}$ which generalizes Dacorogna's well-known theorem on the representation of the quasiconvex envelope of a finite function. The paper will be completed by the calculation of $f^{(q c)}$ in two examples.
\end{abstract}

Mathematics Subject Classification. 26B25, 26B40, 49J45, 52A20.

Received August 30, 2006. Revised June 19, 2007 and October 23, 2007.

Published online November 1st, 2008.

\section{INTRODUCTION}

\subsection{Nonconvex relaxation of multidimensional control problems}

The present paper opens a series of publications, based on the author's habilitation thesis [42]. Their final goal is to obtain existence and relaxation theorems for multidimensional control problems of Dieudonné-Rashevsky type:

$$
\begin{aligned}
(\mathrm{P}): \quad F(x) & =\int_{\Omega} f_{0}(t, x(t), J x(t)) \mathrm{d} t \longrightarrow \inf ! ; \quad x \in W_{0}^{1, \infty}\left(\Omega, \mathbb{R}^{n}\right) ; \\
J x(t) & =\left(\begin{array}{ccc}
\frac{\partial x_{1}}{\partial t_{1}}(t) & \ldots & \frac{\partial x_{1}}{\partial t_{m}}(t) \\
\vdots & & \vdots \\
\frac{\partial x_{n}}{\partial t_{1}}(t) & \ldots & \frac{\partial x_{n}}{\partial t_{m}}(t)
\end{array}\right) \in \mathrm{K} \subset \mathbb{R}^{n \times m}(\forall) t \in \Omega .
\end{aligned}
$$

\footnotetext{
Keywords and phrases. Unbounded function, quasiconvex function, quasiconvex envelope, Morrey's integral inequality, representation theorem.

1 Brandenburg University of Technology, Cottbus; Department of Mathematics, P.O.B. 1013 44, 03013 Cottbus, Germany. Homepage / e-mail: www.thecitytocome.de; wagner@math.tu-cottbus.de
} 
Here the dimensions are $n \geqslant 1, m \geqslant 2$ while $\Omega \subset \mathbb{R}^{m}$ is the closure of a bounded Lipschitz domain, $\mathrm{K} \subset \mathbb{R}^{n m}$ is a convex body with $\mathfrak{o} \in \operatorname{int}(\mathrm{K})$ and $f_{0}(t, \xi, v): \Omega \times \mathbb{R}^{n} \times \mathrm{K} \rightarrow \mathbb{R}$ is a continuous, in general nonconvex function. Problems of this kind arise e.g. in the description of the torsion of prismatic bars ${ }^{1}$, in optimization problems for convex bodies under geometrical restrictions ${ }^{2}$ and within the framework of image processing ${ }^{3}$. In their papers on underdetermined boundary value problems for nonlinear first-order PDE's from the end of the $90 s^{4}$, Dacorogna and Marcellini arrived at Dieudonné-Rashevsky type problems as well.

As yet, relaxation theorems for multidimensional control problems of this type have been obtained in the case of one variable only $(n=1)^{5}$. In contrast to this situation, the comprehension of control restrictions within problems of image processing with possibly nonconvex integrands ${ }^{6}$, as for example the smoothing of color image data or the determination of the optical flow $^{7}$, requires the extension of the known results to the case $n \geqslant 2$. Then in analogy to the multidimensional Calculus of Variations, one has to look for the quasiconvex relaxation of the problem instead of a convex one.

\subsection{Quasiconvexity and unbounded integrands}

The starting point of the present paper is the question how to understand the notion of quasiconvexity for a continuous function $f(v)$ for which the range of definition is a convex body $\mathrm{K} \subset \mathbb{R}^{n m}$ instead of the whole space $\mathbb{R}^{n m}$. The proof of Ekeland/Témam's relaxation theorem as well as the example [41], p. $241 \mathrm{f}$., Theorem 5, suggest the necessity to extend the function with $+\infty$ to $\mathbb{R}^{n m} \backslash \mathrm{K}$ before the eventual forming of an envelope. For this reason, we consider quasiconvex functions with values in $\overline{\mathbb{R}}=\mathbb{R} \cup\{+\infty\}$ and a convex effective domain. As an appropriate envelope, we define and investigate the lower semicontinuous quasiconvex envelope $f^{(q c)}$. The main result of the present paper is a representation theorem for $f^{(q c)}$ (Thm. 4.1 below) which generalizes Dacorogna's well-known theorem on the representation of the quasiconvex envelope of a finite function. By means of $f^{(q c)}$, the author was able to prove a relaxation theorem for $(\mathrm{P})$ in the vectorial case $n \geqslant 2^{8}$, which will be subject of a subsequent publication.

\subsection{Outline of the paper}

After recalling the generalized notions of convexity, we consider in Section 2 quasiconvex functions with values in $\overline{\mathbb{R}}=\mathbb{R} \cup\{+\infty\}$ (Def. 2.9) and a convex effective domain $\mathrm{K}=\operatorname{dom}(f)$. Then in Morrey's integral inequality

$$
f(v) \leqslant \frac{1}{|\Omega|} \int_{\Omega} f(v+J x(t)) \mathrm{d} t \quad \forall x \in W_{0}^{1, \infty}\left(\Omega, \mathbb{R}^{n}\right),
$$

only those Lipschitz functions $x$ must be admitted for variation which satisfy $v+J x(t) \in \mathrm{K}$ for almost all $t \in \Omega$ (Thm. 2.11). In order to find the adequate generalization of the notion of the quasiconvex envelope, we observe that in the forming of the envelope for a finite-valued function $f$, all admissible quasiconvex functions $g \leqslant f$ must be automatically continuous. Thus we form an envelope $f^{(q c)}$ as the pointwise supremum of all lower semicontinuous, quasiconvex functions $g \leqslant f$ with values in $\overline{\mathbb{R}}$ (Def. 2.14(2)). If $f$ belongs to the class $\mathcal{F}_{K}{ }^{9}$ then $f^{(q c)}$ is the largest lower semicontinuous, quasiconvex function below $f$ (Thm. 2.19). The necessity to extend the integrand in (P) with $+\infty$ to $\mathbb{R}^{n m} \backslash \mathrm{K}$ before the forming of the envelope will be confirmed by two examples.

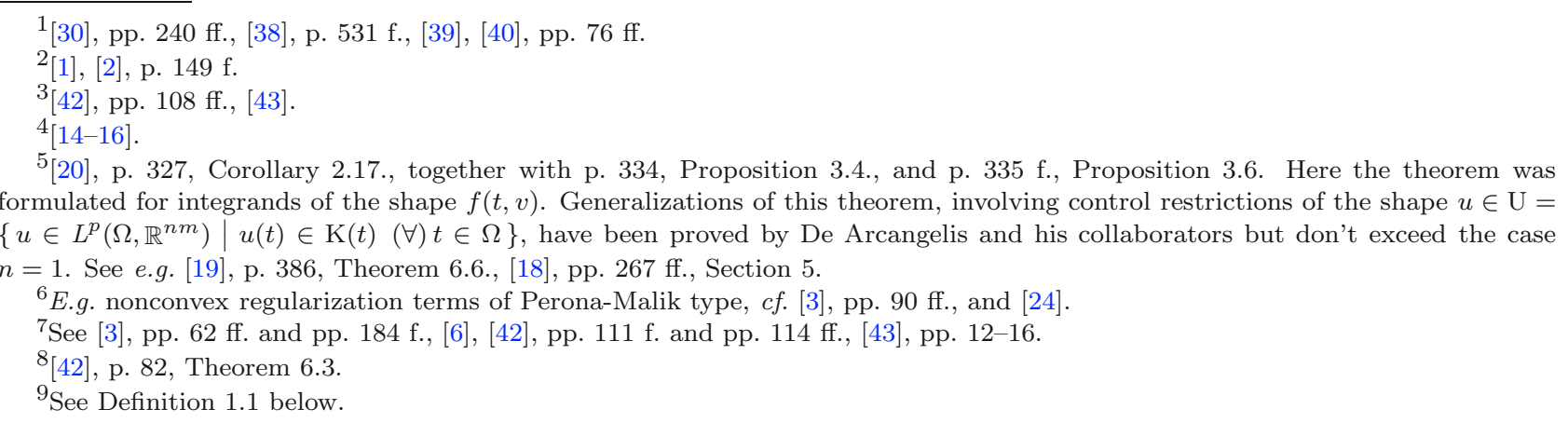


In Section 3, we will clarify the relations between the newly introduced envelope $f^{(q c)}$ and the function $f^{*}$ proposed by Dacorogna/Marcellini ${ }^{10}$ which is defined as

$$
f^{*}(v)=\inf \left\{\frac{1}{|\Omega|} \int_{\Omega} f(v+J x(t)) \mathrm{d} t \mid x \in W_{0}^{1, \infty}\left(\Omega, \mathbb{R}^{n}\right), v+J x(t) \in \mathrm{K}(\forall) t \in \Omega\right\}
$$

in the case $f \in \mathcal{F}_{\mathrm{K}}$. We arrive at the following results: $f^{*}$ and $f^{(q c)}$ coincide in the interior but disagree, in general, on the boundary of K. $f^{*}$ is continuous on int $(\mathrm{K})$ as well as on the relative interior of the faces of $\mathrm{K}$ (Thm. 3.6(1)) but in general not lower semicontinuous while $f^{(q c)}$ arises as the lower semicontinuous envelope of $f^{*}$ (Thm. 3.16 together with Thm. 4.1).

In Section 4, we prove the announced representation theorem for the lower semicontinuous quasiconvex envelope: $f^{(q c)}(v)$ may be represented by (1.4) for $v \in \operatorname{int}(\mathrm{K})$ and by the radial limit

$$
\lim _{v \rightarrow v_{0}, v \in \mathrm{R} \cap \operatorname{int}(\mathrm{K})} f^{*}(v)
$$

along the ray $\mathrm{R}=\overrightarrow{\mathfrak{o} v_{0}}$ for $v_{0} \in \partial \mathrm{K}$ (Thm. 4.1). The proof is based on a continuity relation for $f^{*}$ (Thm. 3.5), which improves a theorem from [14], and the existence and uniformity of the limit (1.5) (Thm. 3.12). The paper will be completed by two examples. Following [41], pp. 242 ff., we define on the four-dimensional cube $\mathrm{K}=[-1,1]^{4}$ a function $f_{0}: \mathrm{K} \rightarrow \mathbb{R}$ for which (after extension with $+\infty$ to $\mathbb{R}^{2 \times 2} \backslash \mathrm{K}$ ) the envelope $f^{(q c)}$ differs from $f^{*}$. The same example shows further that $f^{(q c)}$ as well as $f^{*}$ change discontinuously if $\mathrm{K}$ is approximated in Hausdorff distance.

In Section 5, we have collected some additional facts from measure theory and convex analysis into an appendix.

\subsection{Notations and abbreviations}

Let $k \in\{0,1, \ldots, \infty\}$ and $1 \leqslant p \leqslant \infty$. Then $C^{k}\left(\Omega, \mathbb{R}^{r}\right), L^{p}\left(\Omega, \mathbb{R}^{r}\right)$ and $W^{k, p}\left(\Omega, \mathbb{R}^{r}\right)$ denote the spaces of $r$-dimensional vector functions whose components are $k$-times continuously differentiable, belong to $L^{p}(\Omega)$ or to the Sobolev space of $L^{p}(\Omega)$-functions with weak derivatives up to $k$ th order in $L^{p}(\Omega)$, respectively. In addition, functions within the subspaces $C_{0}^{k}\left(\Omega, \mathbb{R}^{r}\right) \subset C^{k}\left(\Omega, \mathbb{R}^{r}\right)$ are compactly supported while functions within the subspace $W_{0}^{1, \infty}\left(\Omega, \mathbb{R}^{r}\right) \subset W^{1, \infty}\left(\Omega, \mathbb{R}^{r}\right)$ admit a (Lipschitz-) continuous representative ${ }^{11}$ with zero boundary values. The symbols $x_{t_{j}}$ and $\partial x / \partial t_{j}$ may denote the classical as well as the weak partial derivative of $x$ by $t_{j}$. The first Baire function class $\mathcal{B}^{1}(K, \mathbb{R})$ on a compact range of definition $K \subset \mathbb{R}^{n m}$ consists of all finite functions arising as limits of everywhere pointwise convergent sequences of functions $f^{K} \in C^{0}(\mathrm{~K}, \mathbb{R})$; the (finite) limit functions of everywhere pointwise convergent sequences of functions $f^{K} \in \mathcal{B}^{1}(\mathrm{~K}, \mathbb{R})$ form the second Baire class $\mathcal{B}^{2}(\mathrm{~K}, \mathbb{R})$ and so on ${ }^{12}$.

We denote by int (A), ri (A), $\partial \mathrm{A}, \mathrm{rb}(\mathrm{A}), \mathrm{cl}(\mathrm{A}), \mathrm{co}(\mathrm{A})$ and $|\mathrm{A}|$ the interior, relative interior, boundary, relative boundary, closure, the convex hull and the $r$-dimensional Lebesgue measure of the set $\mathrm{A} \subseteq \mathbb{R}^{r}$, respectively. $\mathbb{1}_{\mathrm{A}}: \quad \mathbb{R}^{r} \rightarrow \mathbb{R}$ with $\mathbb{1}_{\mathrm{A}}(t)=1 \Longleftrightarrow t \in \mathrm{A}$ and $\mathbb{1}_{\mathrm{A}}(t)=0 \Longleftrightarrow t \notin \mathrm{A}$ is the characteristic function of the set $\mathrm{A} \subseteq \mathbb{R}^{r}$. We set $\overline{\mathbb{R}}=\mathbb{R} \cup\{+\infty\}$ and equip $\overline{\mathbb{R}}$ with the natural topological and order structures where $+\infty$ is the greatest element. Throughout the whole paper, we consider only proper functions $f: \mathbb{R}^{n m} \rightarrow \overline{\mathbb{R}}$, assuming that $\operatorname{dom}(f)=\left\{v \in \mathbb{R}^{n m} \mid f(v)<+\infty\right\}$ is always nonempty. The restriction of the function $f$ to the subset A of its range of definition is denoted by $f \mid$ A.

Definition 1.1 (function class $\left.\mathcal{F}_{K}\right)$. Let $K \subset \mathbb{R}^{n m}$ be a given convex body with $\mathfrak{o} \in$ int $(\mathrm{K})$. We say that a function $f: \mathbb{R}^{n m} \rightarrow \overline{\mathbb{R}}$ belongs to the class $\mathcal{F}_{K}$ iff $f \mid \mathrm{K} \in C^{0}(\mathrm{~K}, \mathbb{R})$ and $f \mid\left(\mathbb{R}^{n m} \backslash \mathrm{K}\right) \equiv+\infty$.

\footnotetext{
${ }^{10}$ See [14], p. 27, Theorem 7.2., and Definition 3.1. below.

11 [22], p. 131, Theorem 5.

${ }^{12}$ See [7], pp. $393 \mathrm{ff} .$, and the overview in [32], pp. 148-151.
} 
Consequently, any function $f \in \mathcal{F}_{\mathrm{K}}$ is bounded and uniformly continuous on $\mathrm{K}$, and the class $\mathcal{F}_{\mathrm{K}}$ and the Banach space $C^{0}(\mathrm{~K}, \mathbb{R})$ are isomorphic and isometric.

A convex body $\mathrm{K} \subset \mathbb{R}^{n m}$ will be understood as a convex compact set with nonempty interior ${ }^{13}$. A point $v \in \mathrm{K}$ is called extremal point of $\mathrm{K}$ iff from $v=\lambda^{\prime} v^{\prime}+\lambda^{\prime \prime} v^{\prime \prime}, \lambda^{\prime}, \lambda^{\prime \prime}>0, \lambda^{\prime}+\lambda^{\prime \prime}=1, v^{\prime}, v^{\prime \prime} \in \mathrm{K}$ it follows $v^{\prime}=v^{\prime \prime}=v$. The set of all extremal points of $\mathrm{K}$ is denoted by ext $(\mathrm{K})$. For a convex body, ext $(\mathrm{K})$ is always nonempty. A convex subset $\Phi \subseteq \mathrm{K}$ is called a face of $\mathrm{K}$ iff from $v \in \Phi$ and $v=\lambda^{\prime} v^{\prime}+\lambda^{\prime \prime} v^{\prime \prime}, \lambda^{\prime}, \lambda^{\prime \prime}>0, \lambda^{\prime}+\lambda^{\prime \prime}=1, v^{\prime}, v^{\prime \prime} \in \mathrm{K}$ it follows: $v^{\prime}, v^{\prime \prime} \in \bar{\Phi}^{14}$. K itself as well as $\varnothing$ will be regarded as improper faces. All (nonempty) faces of a convex body are compact sets. The dimension $k$ of a face is the dimension of its affine hull; we define $\operatorname{Dim}(\varnothing)=(-1)$. Thus the null-dimensional faces of $\mathrm{K}$ are precisely the singletons $\{x\}, x \in \operatorname{ext}(\mathrm{K})$. A polytope is a set which arises as the convex hull of finitely many points. Consequently, every polytope is compact, and from $\mathrm{P}=$ co $\left(\left\{v_{1}, \ldots, v_{s}\right\}\right)$ it follows that ext $(\mathrm{P}) \subseteq\left\{v_{1}, \ldots, v_{s}\right\}$. The Hausdorff distance of two nonempty, compact sets $\mathrm{A}^{\prime}, \mathrm{A}^{\prime \prime} \subset \mathbb{R}^{n m}$ is defined by $\mathcal{H}\left(\mathrm{A}^{\prime}, \mathrm{A}^{\prime \prime}\right)=\operatorname{Max}\left(\operatorname{Max}_{v^{\prime} \in \mathrm{A}^{\prime}} \operatorname{Dist}\left(v^{\prime}, \mathrm{A}^{\prime \prime}\right), \operatorname{Max}_{v^{\prime \prime} \in \mathrm{A}^{\prime \prime}} \operatorname{Dist}\left(v^{\prime \prime}, \mathrm{A}^{\prime}\right)\right)^{15}$.

We close this subsection with three nonstandard notations. " $\left\{x^{N}\right\}, \mathrm{A}^{\prime}$ denotes a sequence $\left\{x^{N}\right\}$ with members $x^{N} \in \mathrm{A}$. If $\mathrm{A} \subseteq \mathbb{R}^{r}$ then the abbreviation " $(\forall) t \in \mathrm{A}$ " has to be read as "for almost all $t \in \mathrm{A}$ " resp. "for all $t \in \mathrm{A}$ except a $r$-dimensional Lebesgue null set". The symbol $\mathfrak{o}$ denotes, depending on the context, the zero element resp. the zero function of the underlying space.

\section{THE LOWER SEMICONTINUOUS QUASICONVEX ENVELOPE}

\subsection{Generalized notions of convexity}

We start with a recall of the generalized convexity notions to be used in the present paper ${ }^{16}$. Among these notions, the concept of quasiconvexity introduced in 1952 by Morrey ${ }^{17}$ as a necessary and sufficient condition for the weak (resp. weak*-) lower semicontinuity of objective functionals in the multidimensional calculus of variations ${ }^{18}$ takes a special position. As distinct from the other convexity notions, quasiconvexity has to be defined through an integral inequality; in consequence of this fact, this property can be characterized only through countably many algebraic variational inequalities ${ }^{19}$ and cannot be localized ${ }^{20}$. Furthermore, for the classification of quasiconvexity within the series of the generalized convexity notions (convexity and polyconvexity as sufficient, rank one convexity and separate convexity as necessary conditions for it), the restriction to functions with finite values is essential ${ }^{21}$.

Definition 2.1 (Convexity notions for functions with values in $\overline{\mathbb{R}})^{22}$.

(1) (Convex function) A function $f: \mathbb{R}^{n m} \rightarrow \overline{\mathbb{R}}$ is said to be convex if Jensen's inequality is satisfied for every $v^{\prime}, v^{\prime \prime} \in \mathbb{R}^{n m}: f\left(\lambda^{\prime} v^{\prime}+\lambda^{\prime \prime} v^{\prime \prime}\right) \leqslant \lambda^{\prime} f\left(v^{\prime}\right)+\lambda^{\prime \prime} f\left(v^{\prime \prime}\right) \quad \forall \lambda^{\prime}, \lambda^{\prime \prime} \geqslant 0, \lambda^{\prime}+\lambda^{\prime \prime}=1$.

(2) (Rank one convex function) A function $f: \mathbb{R}^{n m} \rightarrow \overline{\mathbb{R}}$ is said to be rank one convex if Jensen's inequality is satisfied in any rank one direction: for every $v^{\prime}, v^{\prime \prime} \in \mathbb{R}^{n m}$ (considered as $(n, m)$-matrices) it holds: $\operatorname{Rg}\left(v^{\prime}-v^{\prime \prime}\right) \leqslant 1 \Longrightarrow f\left(\lambda^{\prime} v^{\prime}+\lambda^{\prime \prime} v^{\prime \prime}\right) \leqslant \lambda^{\prime} f\left(v^{\prime}\right)+\lambda^{\prime \prime} f\left(v^{\prime \prime}\right) \quad \forall \lambda^{\prime}, \lambda^{\prime \prime} \geqslant 0, \lambda^{\prime}+\lambda^{\prime \prime}=1$.

(3) (Separately convex function) A function $f: \mathbb{R}^{n m} \rightarrow \overline{\mathbb{R}}$ is said to be separately convex if it is convex in every variable $v_{i j}$ while the other arguments are fixed.

These properties have in common that they are conserved if a pointwise supremum is formed. Thus the following generalized convex envelopes are well-defined:

\footnotetext{
${ }^{13}$ We follow $[5,35]$.

${ }^{14}$ We dispense with the distinction between "facets" and "faces", $c f$. [5], p. 30.

15 [35], p. 48.

${ }^{16}$ We follow [12], pp. $155 \mathrm{ff}$. and $265 \mathrm{ff}$.

17 [31], p. 28, Definition 2.2 .

18 [12], p. 368, Theorem 8.1., p. 378, Theorem 8.8., and p. 382, Theorem 8.11.

19 [8], p. 13 f., Theorem 2.1 .

20 [26], p. 6, Proposition 2, and p. 8, Theorem 1, in further development of the example from [37].

${ }^{21}$ See [16], p. 132 f., Remark 5.24. (iv), in the context of envelopes for indicator functions.

${ }^{22}$ In the present paper, the concept of polyconvexity will not be used.
} 
Definition 2.2 (Generalized convex envelopes). Let $f: \mathbb{R}^{n m} \rightarrow \overline{\mathbb{R}}$ be a function bounded from below.

(1) (Convex envelope $f^{c}$ ) The convex envelope $f^{c}: \mathbb{R}^{n m} \rightarrow \overline{\mathbb{R}}$ of $f$ is defined by

$$
f^{c}(v)=\sup \left\{g(v) \mid g: \mathbb{R}^{n m} \rightarrow \overline{\mathbb{R}} \text { convex, } g(v) \leqslant f(v) \forall v \in \mathbb{R}^{n m}\right\} .
$$

(2) (Rank one convex envelope $f^{r c}$ ) The rank one convex envelope $f^{r c}: \mathbb{R}^{n m} \rightarrow \overline{\mathbb{R}}$ of $f$ is defined by

$$
f^{r c}(v)=\sup \left\{g(v) \mid g: \mathbb{R}^{n m} \rightarrow \overline{\mathbb{R}} \text { rank one convex, } g(v) \leqslant f(v) \forall v \in \mathbb{R}^{n m}\right\} .
$$

Theorem 2.3 (Continuity of separately convex functions) ${ }^{23}$. Any separately convex function $f: \mathbb{R}^{n m}$ $\rightarrow \overline{\mathbb{R}}$ is continuous on int $(\operatorname{dom}(f))$ (even locally Lipschitz continuous). In particular, every rank one convex function $f: \mathbb{R}^{n m} \rightarrow \overline{\mathbb{R}}$ is continuous on $\operatorname{int}(\operatorname{dom}(f))$.

As mentioned above, the concept of quasiconvexity is applied to functions with finite values almost overall in the literature. The extensions of Definitions 2.4 and 2.5 to functions with values in $\overline{\mathbb{R}}$ will be given in Sections 2.2 and 2.3 below (Defs. 2.9 resp. 2.14).

Definition 2.4 (Quasiconvex function with values in $\mathbb{R})^{24}$. A function $f: \mathbb{R}^{n m} \rightarrow \mathbb{R}$ is said to be quasiconvex if it is Borel measurable, bounded from below on every bounded subset of $\mathbb{R}^{n m}$, and satisfies Morrey's integral inequality ${ }^{25}$ for all $v \in \mathbb{R}^{n m}$ :

$$
f(v) \leqslant \frac{1}{|\Omega|} \int_{\Omega} f(v+J x(t)) \mathrm{d} t \quad \forall x \in W_{0}^{1, \infty}\left(\Omega, \mathbb{R}^{n}\right)
$$

or equivalently

$$
f(v)=\inf \left\{\frac{1}{|\Omega|} \int_{\Omega} f(v+J x(t)) \mathrm{d} t \mid x \in W_{0}^{1, \infty}\left(\Omega, \mathbb{R}^{n}\right)\right\} .
$$

Here $\Omega \subset \mathbb{R}^{m}$ is the closure of a bounded strongly Lipschitz domain.

Definition 2.5 (Quasiconvex envelope $f^{q c}$ for functions with values in $\mathbb{R}$ ). Let $f: \mathbb{R}^{n m} \rightarrow \mathbb{R}$ be a function bounded from below. Its quasiconvex envelope $f^{q c}: \mathbb{R}^{n m} \rightarrow \mathbb{R}$ is defined by

$$
f^{q c}(v)=\sup \left\{g(v) \mid g: \mathbb{R}^{n m} \rightarrow \mathbb{R} \text { quasiconvex, } g(v) \leqslant f(v) \forall v \in \mathbb{R}^{n m}\right\} .
$$

Theorem 2.6 (Relations between the generalized convexity notions) ${ }^{26}$.

(1) (Functions with values in $\overline{\mathbb{R}}$ ) For any function $f: \mathbb{R}^{n m} \rightarrow \overline{\mathbb{R}}$, we have the implications: $f$ convex $\Longrightarrow$ $f$ rank one convex $\Longrightarrow f$ separately convex. If $n=1$ or $m=1$ then we have the equivalence: $f$ convex $\Longleftrightarrow f$ rank one convex.

(2) (Functions with values in $\mathbb{R}$ ) For any function $f: \mathbb{R}^{n m} \rightarrow \mathbb{R}$, we have the implications: $f$ convex $\Longrightarrow$ $f$ quasiconvex $\Longrightarrow f$ rank one convex $\Longrightarrow f$ separately convex. If $n=1$ or $m=1$ then we have the equivalences $f$ convex $\Longleftrightarrow f$ quasiconvex $\Longleftrightarrow f$ rank one convex.

$23[12]$, p. 47 , Theorem 2.31 .

${ }^{24}$ Slightly modified after [12], p. 156 f., Definition 5.1. (ii). The propositions about quasiconvex functions with values in $\mathbb{R}$ cited below will not be altered by this change. In the finite-dimensional optimization, the notion "quasiconvex function" is used in a completely different sense. There it is understood as a function $f$ with convex level sets: $f\left(\lambda^{\prime} v^{\prime}+\lambda^{\prime \prime} v^{\prime \prime}\right) \leqslant \operatorname{Max}\left(f\left(v^{\prime}\right), f\left(v^{\prime \prime}\right)\right)$ $\forall v^{\prime}, v^{\prime \prime} \in \mathbb{R}^{m} \forall \lambda^{\prime}, \lambda^{\prime \prime} \geqslant 0, \lambda^{\prime}+\lambda^{\prime \prime}=1$ (see [10], p. 87).

${ }^{25}$ In the following, we rely upon inequality $(2.5)$ so often that it seems reasonable to coin this notion. A confusion of Morrey's integral inequality with the Morrey inequality from the theory of Sobolev spaces (see [22], p. 143, Thm. 3) is not to be feared.

$26[12]$, p. 159 , Theorem 5.3 , (i) and (ii). 
An immediate consequence of Theorem 2.6. is:

Theorem 2.7 (Relations between the envelopes).

(1) For any function $f: \mathbb{R}^{n m} \rightarrow \overline{\mathbb{R}}$ bounded from below, the following inequalities hold:

$$
f^{c}(v) \leqslant f^{r c}(v) \leqslant f(v) \quad \forall v \in \mathbb{R}^{n m} .
$$

(2) For any function $f: \mathbb{R}^{n m} \rightarrow \mathbb{R}$ bounded from below, the following inequalities hold:

$$
f^{c}(v) \leqslant f^{q c}(v) \leqslant f^{r c}(v) \leqslant f(v) \quad \forall v \in \mathbb{R}^{n m}
$$

For the quasiconvex envelope, we have Dacorogna's representation theorem:

Theorem 2.8 (Representation of $f^{q c}$ for functions with values in $\left.\mathbb{R}\right)^{27}$. Assume that $f: \mathbb{R}^{n m} \rightarrow \mathbb{R}$ is Borel measurable, bounded from below on $\mathbb{R}^{n m}$ and bounded from above on every compact subset of $\mathbb{R}^{n m}$. Then $f^{q c}(v)$ admits the representation

$$
f^{q c}(v)=\inf \left\{\frac{1}{|\Omega|} \int_{\Omega} f(v+J x(t)) \mathrm{d} t \mid x \in W_{0}^{1, \infty}\left(\Omega, \mathbb{R}^{n}\right)\right\}
$$

for all $v \in \mathbb{R}^{n m}$ where $\Omega \subset \mathbb{R}^{m}$ is the closure of a bounded strongly Lipschitz domain.

\subsection{Quasiconvex functions which can take the value $+\infty$}

In this section, we turn to the investigation of quasiconvex functions with values in $\overline{\mathbb{R}}^{28}$. Following [41], we start with the extension of Definition 2.4.

Definition 2.9 (Quasiconvex function with values in $\overline{\mathbb{R}})^{29}$. A function $f: \mathbb{R}^{n m} \rightarrow \overline{\mathbb{R}}$ with the following properties is said to be quasiconvex:

(1) $\operatorname{dom}(f) \subseteq \mathbb{R}^{n m}$ is a (nonempty) Borel set;

(2) $f \mid \operatorname{dom}(f)$ is Borel measurable and bounded from below on every bounded subset of dom $(f)$;

(3) for all $v \in \mathbb{R}^{n m}, f$ satisfies Morrey's integral inequality (see Def. 2.4).

Remarks. (a) For a finite function $f: \mathbb{R}^{n m} \rightarrow \mathbb{R}$, Definitions 2.9 and 2.4 coincide. For this reason, in the following the notion "quasiconvex function" will be understood always in the sense of Definition 2.9.

(b) We agree with the convention that the integral $\int_{\mathrm{A}}(+\infty) \mathrm{d} t$ takes the values zero or $+\infty$ if either $\mathrm{A} \subseteq \mathbb{R}^{m}$ is an $m$-dimensional Lebesgue null set or has positive measure.

(c) The conditions (1) and (2) guarantee that the compositions $f(v+u(\cdot))$ and $\mathbb{1}_{\operatorname{dom}(f)}(v+u(\cdot))$ with $v \in \mathbb{R}^{n m}$ and $u \in L^{\infty}\left(\Omega, \mathbb{R}^{n m}\right)$ are Borel measurable ${ }^{30}$. If $x \in W_{0}^{1, \infty}\left(\Omega, \mathbb{R}^{n}\right)$ then $v+J x(t)$ takes values within a bounded set $\mathrm{B} \subset \mathbb{R}^{n m}$, and the Lebesgue integral

$$
\begin{aligned}
\int_{\Omega} f(v+J x(t)) \mathrm{d} t= & \int_{\Omega}\left(1-\mathbb{1}_{\operatorname{dom}(f)}(v+J x(t))\right) \cdot(+\infty) \mathrm{d} t \\
& +\int_{\Omega} \mathbb{1}_{\operatorname{dom}(f)}(v+J x(t)) \cdot\left(f^{+}(v+J x(t))-f^{-}(v+J x(t))\right) \mathrm{d} t
\end{aligned}
$$

exists as element of $\overline{\mathbb{R}}$ since $\mathbb{1}_{\operatorname{dom}(f)}(v+J x(\cdot)) \cdot f^{-}(v+J x(\cdot))$ is bounded from below in consequence of $(2)$. Note that the values of the integrand $f$ cannot be changed even on a Lebesgue null set of $\mathbb{R}^{n m}$.

\footnotetext{
27 [12], p. 271, Theorem 6.9.; first proven in [11], p. 108, Theorem 5, in a special case.

${ }^{28}$ To the best of the author's knowledge, such functions were considered up to now only in $[4,13,25,41,42]$.

29 [41], p. 237, Definition 5, as specification of [4], p. 228, Definition 2.1., in the case $p=+\infty$. The definition has been changed in the same way as Definition 2.4.

$30[41]$, p. 238 .
} 
The basic properties of quasiconvex functions with values in $\overline{\mathbb{R}}$ will be collected in the following theorems with special attention to the case where $\operatorname{dom}(f)=\mathrm{K}$ is a convex body ${ }^{31}$.

Lemma 2.10 (Operations with quasiconvex functions with values in $\overline{\mathbb{R}}$ ).

(1) Together with $g_{1}, g_{2}: \mathbb{R}^{n m} \rightarrow \overline{\mathbb{R}}$, any nonnegative linear combination is quasiconvex.

(2) Together with $g_{1}, g_{2}: \mathbb{R}^{n m} \rightarrow \overline{\mathbb{R}}$, the function $\operatorname{Max}\left(g_{1}, g_{2}\right)$ is quasiconvex. In particular, every function $\operatorname{Max}\left(g_{1}, c\right), c \in \mathbb{R}$, is quasiconvex together with $g_{1}$.

(3) Let $v_{0} \in \mathbb{R}^{n m}$ and $\mu>0$ be given. Then the function $h(v)=g\left(v_{0}+\mu v\right)$ is quasiconvex together with $g(v): \mathbb{R}^{n m} \rightarrow \overline{\mathbb{R}}$.

Theorem 2.11 (Morrey's integral inequality for functions with $\operatorname{dom}(f)=\mathrm{K})^{32}$. Let a convex body $\mathrm{K} \subset \mathbb{R}^{n m}$ and a function $f: \mathbb{R}^{n m} \rightarrow \overline{\mathbb{R}}$ with $\operatorname{dom}(f)=\mathrm{K}$ be given. Assume that $f \mid \mathrm{K}$ is measurable and bounded. Then the following assertions hold:

(1) For all $v \in \mathbb{R}^{n m} \backslash \mathrm{K}$, Morrey's integral inequality holds in the form $+\infty \leqslant+\infty$.

(2) $f$ satisfies Morrey's integral inequality in a point $v \in \mathrm{K}$ iff

$$
f(v)=\inf \left\{\frac{1}{|\Omega|} \int_{\Omega} f(v+J x(t)) \mathrm{d} t \mid x \in W_{0}^{1, \infty}\left(\Omega, \mathbb{R}^{n}\right), v+J x(t) \in \mathrm{K}(\forall) t \in \Omega\right\} .
$$

(3) Let $\Phi \subseteq \mathrm{K}$ be a $k$-dimensional face of $\mathrm{K}, 0 \leqslant k \leqslant n m . \quad f$ satisfies Morrey's integral inequality in a point $v \in \Phi$ iff

$$
f(v)=\inf \left\{\frac{1}{|\Omega|} \int_{\Omega} f(v+J x(t)) \mathrm{d} t \mid x \in W_{0}^{1, \infty}\left(\Omega, \mathbb{R}^{n}\right), v+J x(t) \in \Phi(\forall) t \in \Omega\right\} .
$$

Theorem 2.12 (Rank one convexity and continuity of quasiconvex functions with dom $(f)=\mathrm{K}$ ). Given a convex body $\mathrm{K} \subset \mathbb{R}^{n m}$ and a function $f: \mathbb{R}^{n m} \rightarrow \overline{\mathbb{R}}$ with $\operatorname{dom}(f)=\mathrm{K}$. Assume that $f$ is quasiconvex and $f \mid \mathrm{K}$ is bounded. Then the restriction $f \mid \operatorname{int}(\mathrm{K})$ is rank one convex and continuous.

In the following assertion we provide some important examples of quasiconvex functions with values in $\overline{\mathbb{R}}$ : indicator functions $\varrho_{\mathrm{C}}$ of closed convex sets, lower semicontinuous convex functions having a convex body as effective domain and being bounded on it, and functions of the shape $\left(1+\varrho_{\mathrm{C}}\right) \cdot f$ where $f$ is finite and quasiconvex.

Lemma 2.13 (Examples of quasiconvex functions with values in $\overline{\mathbb{R}}$ ).

(1) For any nonempty, closed, convex set $\mathrm{C} \subseteq \mathbb{R}^{n m}$, the indicator function $\varrho_{\mathrm{C}}: \mathbb{R}^{n m} \rightarrow \overline{\mathbb{R}}$ defined by

$$
\varrho_{\mathrm{C}}(v)=\left\{\begin{array}{c|l}
0 & \mid v \in \mathrm{C} \\
+\infty & v \in \mathbb{R}^{n m} \backslash \mathrm{C}
\end{array}\right.
$$

is quasiconvex and lower semicontinuous.

(2) Let a lower semicontinuous, convex function $f: \mathbb{R}^{n m} \rightarrow \overline{\mathbb{R}}$ be given. Assume that $\operatorname{dom}(f)=\mathrm{K}$ is a convex body and $f \mid \operatorname{dom}(f)$ is bounded. Then $f$ is quasiconvex.

(3) Let a quasiconvex (and continuous) function $f: \mathbb{R}^{n m} \rightarrow \mathbb{R}$ and a nonempty, closed, convex set $\mathrm{C} \subseteq \mathbb{R}^{n m}$ be given. The the function $g: \mathbb{R}^{n m} \rightarrow \overline{\mathbb{R}}$ defined by

$$
g(v)=\left\{\begin{array}{l|l}
f(v) & \mid v \in \mathrm{C} \\
+\infty & \mid v \in \mathbb{R}^{n m} \backslash \mathrm{C}
\end{array}\right.
$$

is quasiconvex and lower semicontinuous.

\footnotetext{
${ }^{31}$ The generalization of the related propositions to compact, nonconvex sets $\operatorname{dom}(f)=\mathrm{A}$ is not the subject of the present investigation. For that one would need an appropriate notion of the quasiconvex hull of a set (see [16], p. 132, Def. 5.23, [17], p. 108, Def. 3.1, (ii), and p. 114, Def. 5.1, and [44], p. 669, Def. 2.3) as well as further knowledge about the quasiconvex extremal structure of A (see [17], pp. $122 \mathrm{ff} .,[28,29,44,45]$ ).

32 [41], p. 238, Theorem 2, i)-iii).
} 
Proof of Lemma 2.10. (1) is obvious.

(2) If $g_{1}, g_{2}$ are quasiconvex functions then the set $\operatorname{dom}\left(\operatorname{Max}\left(g_{1}, g_{2}\right)\right)=\operatorname{dom}\left(g_{1}\right) \cap \operatorname{dom}\left(g_{2}\right)$ is measurable. Together with $g_{1} \mid \operatorname{dom}\left(g_{1}\right)$ and $g_{2} \mid \operatorname{dom}\left(g_{2}\right)$, the restriction of $\operatorname{Max}\left(g_{1}, g_{2}\right)=\frac{1}{2}\left(g_{1}+g_{2}+\left|g_{1}-g_{2}\right|\right)$ to its effective domain is measurable and bounded from below on every bounded subset of the effective domain. Choosing $v \in \mathbb{R}^{n m}$ and $x \in W_{0}^{1, \infty}\left(\Omega, \mathbb{R}^{n}\right)$, we deduce

$$
\begin{aligned}
& g_{1}(v) \leqslant \frac{1}{|\Omega|} \int_{\Omega} g_{1}(v+J x(t)) \mathrm{d} t \leqslant \frac{1}{|\Omega|} \int_{\Omega} \operatorname{Max}\left(g_{1}, g_{2}\right)(v+J x(t)) \mathrm{d} t ; \\
& g_{2}(v) \leqslant \frac{1}{|\Omega|} \int_{\Omega} g_{2}(v+J x(t)) \mathrm{d} t \leqslant \frac{1}{|\Omega|} \int_{\Omega} \operatorname{Max}\left(g_{1}, g_{2}\right)(v+J x(t)) \mathrm{d} t \Longrightarrow \\
& \operatorname{Max}\left(g_{1}, g_{2}\right)(v) \leqslant \frac{1}{|\Omega|} \int_{\Omega} \operatorname{Max}\left(g_{1}, g_{2}\right)(v+J x(t)) \mathrm{d} t .
\end{aligned}
$$

(3) Together with $\operatorname{dom}(g), \operatorname{dom}(h)=\left\{v \in \mathbb{R}^{n m} \mid v_{0}+\mu v \in \operatorname{dom}(g)\right\}$ is a Borel set. Choose now a Borel set $\mathrm{A} \subseteq \mathbb{R}$. Then it holds:

$$
\begin{aligned}
h^{-1}(\mathrm{~A})=\left\{v \in \mathbb{R}^{n m} \mid h(v) \in \mathrm{A}\right\} & =\left\{v \in \mathbb{R}^{n m} \mid g(\widetilde{v}) \in \mathrm{A}, \widetilde{v}=v_{0}+\mu v\right\} \\
& =\left\{v \in \mathbb{R}^{n m} \mid v=\left(\widetilde{v}-v_{0}\right) / \mu, \widetilde{v} \in g^{-1}(\mathrm{~A})\right\}=g^{-1}(\mathrm{~A}) \cdot 1 / \mu-v_{0} / \mu
\end{aligned}
$$

Thus $h^{-1}(\mathrm{~A})$ is a Borel set of $\mathbb{R}^{n m}$ together with $g^{-1}(\mathrm{~A})$, and the function $h \mid \operatorname{dom}(h)$ is measurable. Further, on every bounded subset $\mathrm{B} \subseteq \operatorname{dom}(h)$ with $|v| \leqslant R$ for all $v \in \mathrm{B}$, the values $h(v)=g\left(v_{0}+\mu v\right)$ are bounded from below since $g$ is bounded from below on the bounded subset of the points $\left(v_{0}+\mu v\right) \in \operatorname{dom}(g)$ with $\left|v_{0}+\mu v\right| \leqslant\left|v_{0}\right|+\mu R$. Finally, for all $v \in \mathbb{R}^{n m}$ it holds:

$h(v)=g\left(v_{0}+\mu v\right) \leqslant \frac{1}{|\Omega|} \int_{\Omega} g\left(v_{0}+\mu v+\mu J x(t)\right) \mathrm{d} t=\frac{1}{|\Omega|} \int_{\Omega} h(v+J x(t)) \mathrm{d} t \quad \forall x \in W_{0}^{1, \infty}\left(\Omega, \mathbb{R}^{n}\right)$,

wherein, particularly, the last integral always exists.

Proof of Theorem 2.12. The validity of Jensen's inequality along rank one directions was proven in [14], p. 33 f., Proof of (7.40). Consequently, the function $h: \mathbb{R}^{n m} \rightarrow \overline{\mathbb{R}}$ defined by

$$
h(v)=\left\{\begin{array}{l}
f(v) \mid v \in \operatorname{int}(\mathrm{K}) \\
+\infty \mid v \in \partial \mathrm{K} \cup\left(\mathbb{R}^{n m} \backslash \mathrm{K}\right)
\end{array}\right.
$$

is rank one convex and, by Theorem 2.3, continuous on int $(\mathrm{K})$.

Proof of Lemma 2.13.

(1) The lower semicontinuous function $\varrho_{\mathrm{C}}$ satisfies the conditions (1) and (2) from Definition 2.9. Since it holds that

$$
\int_{\Omega} \varrho_{\mathrm{C}}(v+J x(t)) \mathrm{d} t=\left\{\begin{array}{c|l}
0 & v+J x(t) \in \mathrm{C}(\forall) t \in \Omega \\
+\infty & v+J x(t) \in \mathbb{R}^{n m} \backslash \mathrm{C} \text { for a } t \text {-set of positive measure }
\end{array}\right.
$$

for arbitrary $x \in W_{0}^{1, \infty}\left(\Omega, \mathbb{R}^{n}\right)$, Morrey's integral inequality is satisfied for all $v \in \mathrm{C}$. Since $\mathrm{C}$ is closed and convex, we find in the case $v \notin \mathrm{C}$ in analogy to [41], p. 238 f., Proof of Theorem 2, (i), that $v+J x(t)$ belongs to $\mathbb{R}^{n m} \backslash \mathrm{C}$ on a $t$-set of positive measure for arbitrary $x \in W_{0}^{1, \infty}\left(\Omega, \mathbb{R}^{n}\right)$. Then the integral takes the value $+\infty$, and Morrey's integral inequality remains valid as well. 
(2) By assumption, dom $(f)$ is compact and thus a Borel set. Together with $f, f \mid \operatorname{dom}(f)$ is lower semicontinuous and, consequently, measurable. Since its boundedness was assumed, the conditions (1) and (2) from Definition 2.9 are satisfied. Let $v \in \operatorname{dom}(f)$ and $x \in W_{0}^{1, \infty}\left(\Omega, \mathbb{R}^{n}\right)$ with $v+J x(t) \in \operatorname{dom}(f)(\forall) t \in \Omega$ be given. Then we deduce from Lemma 5.1(1) together with Jensen's integral inequality for convex functions (see [23], p. 310):

$f(v)=f\left(\left(v_{i j}\right)_{i, j}\right)=f\left(\left(\frac{1}{|\Omega|} \int_{\Omega}\left(v_{i j}+\frac{\partial x_{i}}{\partial t_{j}}(t)\right) \mathrm{d} t\right)_{i, j}\right) \leqslant \frac{1}{|\Omega|} \int_{\Omega} f\left(\left(v_{i j}+\frac{\partial x_{i}}{\partial t_{j}}(t)\right){ }_{i, j}\right) \mathrm{d} t$

and $f$ is quasiconvex by Theorem $2.11(2)$.

(3) Obviously, $g$ is a lower semicontinuous function. Then the assertion follows from (1) and Lemma 2.10(1) and $(2)$.

\subsection{The lower semicontinuous quasiconvex envelope $f^{(q c)}$}

After introducing quasiconvex functions with values in $\overline{\mathbb{R}}$, we turn to the appropriate generalization of the definition of the quasiconvex envelope. Our starting point is the observation that, by Theorems 2.6(2) and 2.3 , finite quasiconvex functions $g: \mathbb{R}^{n m} \rightarrow \mathbb{R}$ are continuous functions from the outset. So we could add this property within Definition 2.5. without changing of the value of $f^{q c}$. Then this definition allows two generalizations. Either we form for $f: \mathbb{R}^{m} \rightarrow \overline{\mathbb{R}}$ an envelope with quasiconvex and finite and thus continuous functions $g$ (Def. 2.14(1)) or we include lower semicontinuous quasiconvex functions with values in $\overline{\mathbb{R}}$ into the formation of the envelope as well (Def. 2.14(2)). In the course of our investigations, we will adopt the second approach. Subsequent to the definitions, the properties of the envelopes for functions $f \in \mathcal{F}_{\mathrm{K}}$ will be closer investigated.

Definition 2.14 (Quasiconvex envelope $f^{q c}$ and lower semicontinuous quasiconvex envelope $f^{(q c)}$ for functions with values in $\overline{\mathbb{R}}$ ). To any function $f: \mathbb{R}^{n m} \rightarrow \overline{\mathbb{R}}$ bounded from below, we define the envelopes $(1) f^{q c}(v)=\sup \left\{g(v) \mid g: \mathbb{R}^{n m} \rightarrow \mathbb{R}\right.$ quasiconvex (and continuous), $\left.g(v) \leqslant f(v) \forall v \in \mathbb{R}^{n m}\right\}$;

$(2) f^{(q c)}(v)=\sup \left\{g(v) \mid g: \mathbb{R}^{n m} \rightarrow \overline{\mathbb{R}}\right.$ quasiconvex and lower semicontinuous, $\left.g(v) \leqslant f(v) \forall v \in \mathbb{R}^{n m}\right\}$.

Remarks. (a) If a function $f$ is bounded from below and takes only values in $\mathbb{R}$ then Definitions 2.5 and $2.14(1)$ coincide. Then the envelope $f^{q c}$ is quasiconvex and continuous, and both envelopes $f^{q c}$ and $f^{(q c)}$ agree since then all quasiconvex functions, which are feasible in (2), are finite (and even continuous) as well.

(b) In view of Theorem 2.16 below, we are justified to call $f^{q c}$ and $f^{(q c)}$ the quasiconvex envelope resp. lower semicontinuous quasiconvex envelope of $f$ while $f: \mathbb{R}^{n m} \rightarrow \overline{\mathbb{R}}$ is bounded from below.

Lemma 2.15. Assume that the functions $f, f_{1}$ and $f_{2}: \mathbb{R}^{n m} \rightarrow \overline{\mathbb{R}}$ are bounded from below. Then it holds:

(1) $f^{q c}(v) \leqslant f^{(q c)}(v) \leqslant f(v) \forall v \in \mathbb{R}^{n m}$.

(2) $f_{1}(v) \leqslant f_{2}(v) \forall v \in \mathbb{R}^{n m} \Longrightarrow f_{1}^{q c}(v) \leqslant f_{2}{ }^{q c}(v) \forall v \in \mathbb{R}^{n m}$.

(3) $f_{1}(v) \leqslant f_{2}(v) \forall v \in \mathbb{R}^{n m} \Longrightarrow f_{1}{ }^{(q c)}(v) \leqslant f_{2}{ }^{(q c)}(v) \forall v \in \mathbb{R}^{n m}$.

Theorem 2.16 (Quasiconvexity of $f^{q c}$ and $f^{(q c)}$ ). Assume that the function $f: \mathbb{R}^{n m} \rightarrow \overline{\mathbb{R}}$ is bounded from below. Then $f^{q c}$ as well as $f^{(q c)}$ are quasiconvex functions in the sense of Definition 2.9.

We emphasize that the following theorems are formulated for functions $f \in \mathcal{F}_{K}$.

Theorem 2.17 (Properties of $f^{c}$ and $f^{q c}$ for $f \in \mathcal{F}_{\mathrm{K}}$ ). For any function $f \in \mathcal{F}_{\mathrm{K}}$ it holds:

(1) $f^{c}(v) \leqslant f^{q c}(v) \leqslant f(v) \forall v \in \mathbb{R}^{n m}$, which implies particularly $f^{q c}(v)=+\infty$ for all $v \in \mathbb{R}^{n m} \backslash \mathrm{K}$ and $f^{q c}(v)=f(v)$ for all $v \in \operatorname{ext}(\mathrm{K})$.

(2) $f^{c}$ is lower semicontinuous and quasiconvex.

(3) $f^{q c}$ is lower semicontinuous and quasiconvex.

(4) $f^{q c} \mid$ int $(\mathrm{K})$ is rank one convex and continuous. 
Theorem 2.18 (Properties of $f^{(q c)}$ for $f \in \mathcal{F}_{\mathrm{K}}$ ). For any function $f \in \mathcal{F}_{\mathrm{K}}$ it holds:

(1) $f^{c}(v) \leqslant f^{(q c)}(v) \leqslant f(v)$ for all $v \in \mathbb{R}^{n m}$, which implies particularly $f^{(q c)}(v)=+\infty$ for all $v \in \mathbb{R}^{n m} \backslash \mathrm{K}$ and $f^{(q c)}(v)=f(v)$ for all $v \in \operatorname{ext}(\mathrm{K})$.

(2) $f^{(q c)}$ is lower semicontinuous and quasiconvex.

(3) $f^{(q c)} \mid \operatorname{int}(\mathrm{K})$ is rank one convex ${ }^{33}$ and continuous.

By Theorem 2.16, $f^{(q c)}$ is (in difference to $f^{q c}$ ) admissible in the process of its own forming. The immediate consequence of this fact is:

Theorem $2.19\left(f^{(q c)}\right.$ for $f \in \mathcal{F}_{\mathrm{K}}$ as the largest quasiconvex, lower semicontinuous function $\left.g \leqslant f\right)$. Let $f \in \mathcal{F}_{\mathrm{K}}$. For any lower semicontinuous, quasiconvex function $g: \mathbb{R}^{n m} \rightarrow \overline{\mathbb{R}}$ from $g(v) \leqslant f(v) \forall v \in \mathbb{R}^{n m}$ it follows that $g(v) \leqslant f^{(q c)}(v) \forall v \in \mathrm{K}$.

Proof of Lemma 2.15. The assertions are obvious.

Proof of Theorem 2.16. Consider a function $f: \mathbb{R}^{n m} \rightarrow \overline{\mathbb{R}}$ which is bounded from below. Since the constant functions are admissible in the forming of $f^{q c}$ as well as of $f^{(q c)}$, both these functions are bounded as well. Moreover, all feasible functions in the forming of $f^{q c}$ and $f^{(q c)}$ are lower semicontinuous, and their epigraphs are closed. Then the epigraphs of $f^{q c}$ and $f^{(q c)}$ as their intersection are closed as well, and $f^{q c}$ and $f^{(q c)}$ are lower semicontinuous functions. Consequently, their effective domains $\operatorname{dom}\left(f^{q c}\right)$ and $\operatorname{dom}\left(f^{(q c)}\right)$ are measurable subsets of $\mathbb{R}^{n m}$ (cf. [21], p. 105, Thm. 4.2), and the restrictions $f^{q c} \mid \operatorname{dom}\left(f^{q c}\right)$ and $f^{(q c)} \mid \operatorname{dom}\left(f^{(q c)}\right)$ are measurable functions. Thus conditions (1) and (2) from Definition 2.9. are satisfied. At last, we have for any quasiconvex, continuous function $g: \mathbb{R}^{n m} \rightarrow \mathbb{R}$ with $g(v) \leqslant f(v) \forall v \in \mathbb{R}^{n m}$, for arbitrary $v \in \mathbb{R}^{n m}$ and $x \in W_{0}^{1, \infty}\left(\Omega, \mathbb{R}^{n}\right):$

$$
\begin{aligned}
& g(v) \leqslant \frac{1}{|\Omega|} \int_{\Omega} g(v+J x(t)) \mathrm{d} t \leqslant \frac{1}{|\Omega|} \int_{\Omega} f^{q c}(v+J x(t)) \mathrm{d} t \Longrightarrow \\
& f^{q c}(v)=\sup _{g} g(v) \leqslant \frac{1}{|\Omega|} \int_{\Omega} f^{q c}(v+J x(t)) \mathrm{d} t
\end{aligned}
$$

and $f^{q c}$ satisfies Morrey's integral inequality. An analogous conclusion holds for $f^{(q c)}$.

Proof of Theorem 2.17. (1) By Theorem 5.7(2), $f^{c}$ can be represented as the pointwise supremum of all affine functions $g: \mathbb{R}^{n m} \rightarrow \mathbb{R}$ with $g \leqslant f$. Since any finite, convex function (and thus, in particular, any affine function) is quasiconvex (Thm. 2.6(2)), any function $g$ which is feasible in the forming of $f^{c}$ is feasible in the forming of $f^{q c}$ as well, and we arrive at $f^{c}(v) \leqslant f^{q c}(v)$ for all $v \in \mathbb{R}^{n m}$. By Theorem $5.7(3),+\infty=f^{c}(v) \leqslant$ $f^{q c}(v)=+\infty$ for all $v \in \mathbb{R}^{n m} \backslash \mathrm{K}$, and by Theorem 5.7(5), we find $f^{c}(v)=f^{q c}(v)=f(v)$ for all $v \in \operatorname{ext}(\mathrm{K})$.

(2) From Theorem 5.7(4) we see that $f^{c} \mid \mathrm{K}$ is bounded together with $f \mid \mathrm{K}$. By Theorem 5.7(1), $f^{c}$ is lower semicontinuous. Then Lemma 2.13(2) implies its quasiconvexity.

(3) By Theorem 2.16, this holds true since $f \in \mathcal{F}_{\mathrm{K}}$ is bounded. In consequence of this fact, assertion (4) follows from Theorem 2.12 .

Proof of Theorem 2.18. (1) By Theorem 2.17(3), $f^{q c}$ is feasible in the forming of $f^{(q c)}$. In view of Theorem 2.16 , (2) is obvious. Then (3) is again a consequence of Theorem 2.12.

Proof of Theorem 2.19. By Theorem 2.18(2), $f^{(q c)}$ is feasible within its own forming.

\subsection{Quasiconvexity and the extension of the integrand in (P) to $\mathbb{R}^{n m} \backslash K$}

The continuous integrand $f_{0}$ within the objective of the control problem $(\mathrm{P})$ is defined on $\mathrm{K}$ only. Thus one can neither decide whether $f_{0}$ is quasiconvex (in the sense of Def. 2.4 or Def. 2.9) nor form a quasiconvex

\footnotetext{
${ }^{33}$ We will prove below (Thm. $\left.4.2(1)\right)$ that $f(q c): \mathbb{R}^{n m} \rightarrow \overline{\mathbb{R}}$ itself is rank one convex.
} 
envelope according to Definitions 2.5 or 2.14. In both cases, $f_{0}$ has to be extended before to the whole space. The question arises whether some finite extension or the infinite extension

$$
f(v)=\left\{\begin{array}{l|l}
f_{0}(v) & \mid v \in \mathrm{K} \\
+\infty & v \in \mathbb{R}^{n m} \backslash \mathrm{K}
\end{array}\right.
$$

to a function $f \in \mathcal{F}_{\mathrm{K}}$ is appropriate. We provide two examples which strongly suggest that one has to prefer the infinite extension. Both are concerned with functions of arguments $v=\left(\begin{array}{ll}a & b \\ c & d\end{array}\right) \in \mathbb{R}^{2 \times 2}$ which will be understood as $(2,2)$-matrices. In the space $\mathbb{R}^{2 \times 2}$, we use the norm $|v|=\left(a^{2}+b^{2}+c^{2}+d^{2}\right)^{1 / 2}$.

In the first example, we consider a continuous, convex function $f_{0}: \mathrm{K} \rightarrow \mathbb{R}$. It is well-known that the possibility of extension of $f_{0}$ to a finite, convex function on the whole space depends on the joint properties of the subdifferentials $\partial f_{0}(\cdot)$ and $\partial \varrho_{\mathrm{K}}(\cdot)^{34}$. Up to now, the conditions which allow the extension of a convex continuous function $f_{0}: \mathrm{K} \rightarrow \mathbb{R}$ to a finite quasiconvex function on the whole space have been investigated in a special case only ${ }^{35}$. We define $\mathrm{K}$ and $f_{0}$ as follows:

Definition 2.20. Let $\mathrm{K}=\left\{v \in \mathbb{R}^{2 \times 2}|| v \mid \leqslant 1\right\}$ be the four-dimensional closed unit ball. By means of the function $g(r):[-1,1] \rightarrow \mathbb{R}$ defined by $g(r)=1-\left(1-r^{2}\right)^{1 / 2}$, we declare $f_{0}: \mathrm{K} \rightarrow \mathbb{R}$ as

$$
f_{0}(v)=g(|v|)=1-\left(1-a^{2}-b^{2}-c^{2}-d^{2}\right)^{1 / 2} .
$$

Theorem 2.21 (Properties of the function $f_{0}$ from Def. 2.20).

(1) $f_{0}$ belongs to $C^{0}(\mathrm{~K}, \mathbb{R}) \cap\left(W^{1,1}(\mathrm{~K}, \mathbb{R}) \backslash W^{1, \infty}(\mathrm{K}, \mathbb{R})\right)$.

(2) $f_{0}$ is a convex function on $\mathrm{K}$.

(3) For any finite, continuous extension $\tilde{f}: \mathbb{R}^{2 \times 2} \rightarrow \mathbb{R}$ of $f_{0}$ to the whole space, there exists some $v \in$ int $(\mathrm{K})$ with $\tilde{f}^{c}(v) \leqslant \tilde{f}^{q c}(v)<f_{0}(v)$.

(4) The infinite extension

$$
f(v)=\left\{\begin{array}{l|l}
f_{0}(v) & \mid v \in \mathrm{K} \\
+\infty & v \in \mathbb{R}^{n m} \backslash \mathrm{K}
\end{array}\right.
$$

belongs to $\mathcal{F}_{\mathrm{K}}$ and is quasiconvex.

Consequently, $f_{0}$ can neither be extended to a finite, convex function on $\mathbb{R}^{2 \times 2}$, nor any of the quasiconvex envelopes $\tilde{f}^{q c}$ is the greatest lower semicontinuous quasiconvex function below the infinite extension $f \in \mathcal{F}_{\mathrm{K}}$ while $f^{(q c)}$ and $f$ coincide.

Our second example is a slight sharpening of [41], p. $241 \mathrm{f}$., Definition 7 and Theorem 5 . It is concerned with a continuous function $f_{0}: \mathrm{K} \rightarrow \mathbb{R}$ with a discontinuous convex envelope $\left(f_{0}\right)^{c}: \mathrm{K} \rightarrow \mathbb{R}$. Consequently, $\left(f_{0}\right)^{c}$ cannot be extended to a finite, quasiconvex function on the whole space.

Definition 2.22 $\mathbf{2}^{36}$. Let the points $v_{1}=\left(\begin{array}{rr}-1 & -1 \\ 0 & 0\end{array}\right), v_{2}=\left(\begin{array}{rr}1 & -1 \\ 0 & 0\end{array}\right)$ and the convex set $\mathrm{C}=\left\{\left(\begin{array}{ll}0 & b \\ c & d\end{array}\right) \in \mathbb{R}^{2 \times 2} \mid b^{2}+c^{2}+\right.$ $\left.d^{2} \leqslant 1\right\}$ be given. Define sets $\mathrm{K}_{1}=\mathrm{co}\left(\left\{v_{1}\right\} \cup \mathrm{C}\right), \mathrm{K}_{2}=\mathrm{co}\left(\left\{v_{2}\right\} \cup \mathrm{C}\right), \mathrm{K}=\mathrm{K}_{1} \cup \mathrm{K}_{2}{ }^{37}$ and $f_{0}: \mathrm{K} \rightarrow \mathbb{R}$ by

$$
f_{0}(v)=\left(a^{2}-1\right)^{2} .
$$

Theorem 2.23 (Properties of the convex body $K$ and the function $f_{0}$ from Def. 2.22) ${ }^{38}$. (1) $\mathrm{K}$ is a closed convex set with $\mathfrak{o}_{4} \in \operatorname{int}(\mathrm{K})$ and $\operatorname{ext}(\mathrm{K})=\left\{v_{1}, v_{2}\right\} \cup\left(\operatorname{ext}(\mathrm{C}) \backslash\left\{\left(\begin{array}{rr}0 & -1 \\ 0 & 0\end{array}\right)\right\}\right)$.

\footnotetext{
$34[36]$, p. 505, Theorem 1.

$35[26]$, p. 5, Lemma 2.

36 [41], p. 241, Definition 7.

37 The idea for the construction of $\mathrm{K}$ can be traced back to [27], p. $698 \mathrm{f}$.

${ }^{38}$ Part 1): [41], p. 241, Lemma 1; Parts 2)-4): [41], p. 241 f., Theorem 5.
} 
(2) $f_{0}$ belongs to $W^{1, \infty}(\mathrm{K}, \mathbb{R})$ and is infinitely differentiable on $\operatorname{int}(\mathrm{K})$.

(3) For all points $\left(\begin{array}{ll}0 & b \\ c & d\end{array}\right) \in \operatorname{ext}(\mathrm{C})$ with $b \neq(-1)$, we have $\left(f_{0}\right)^{c}\left(\begin{array}{ll}0 & b \\ c & d\end{array}\right)=1$ but $\left(f_{0}\right)^{c}\left(\begin{array}{cc}0 & -1 \\ c & d\end{array}\right)=0$. Consequently, $\left(f_{0}\right)^{c}$ has in $\left(\begin{array}{cc}0 & -1 \\ 0 & 0\end{array}\right)$ a point of discontinuity.

(4) Any finite, continuous extension $\tilde{f}: \mathbb{R}^{2 \times 2} \rightarrow \mathbb{R}$ of $f_{0}$ satisfies $\tilde{f}^{q c}\left(\begin{array}{cc}0 & -1 \\ 0 & 0\end{array}\right) \leqslant 0$. Consequently, $\tilde{f}^{c}(v) \leqslant$ $\tilde{f}^{q c}(v)<\left(f_{0}\right)^{c}(v)$ for all points $v \in \operatorname{ext}(\mathrm{K})$ with sufficiently small distance to $\left(\begin{array}{cc}0 & -1 \\ 0 & 0\end{array}\right)$.

(5) The convex envelope $f^{c}$ of the infinite extension

$$
f(v)=\left\{\begin{array}{c|l}
f_{0}(v) & \mid v \in \mathrm{K} \\
+\infty & v \in \mathbb{R}^{n m} \backslash \mathrm{K}
\end{array}\right.
$$

is given through

$$
f^{c}(v)=\left\{\begin{array}{c|l}
\left(f_{0}\right)^{c}(v) & v \in \mathrm{K} \\
+\infty & v \in \mathbb{R}^{n m} \backslash \mathrm{K}
\end{array}\right.
$$

and is a quasiconvex function.

As in the first example, $\left(f_{0}\right)^{c}$ cannot be extended to a finite, convex function on $\mathbb{R}^{2 \times 2}$. Again, none of the quasiconvex envelopes $\widetilde{f}^{q c}$ is the greatest lower semicontinuous quasiconvex function below the infinite extension $f \in \mathcal{F}_{\mathrm{K}}$ since, by (3) and (4), there exist points $v \in \mathrm{K}$ with $\tilde{f}^{q c}(v)<\left(f_{0}\right)^{c}(v)=f^{c}(v) \leqslant f^{(q c)}(v)$.

Proof of Theorem 2.21. (1) and (2) follow after easy computations, see [42], p. 18.

(3) Consider any finite, continuous extension $\widetilde{f}$ of $f_{0}$ to $\mathbb{R}^{2 \times 2}$. After choosing a point $v_{0} \in \mathbb{R}^{2 \times 2}$ with $\left|v_{0}\right|>1$ and $\operatorname{Rg}\left(v_{0}\right)=1$, we consider the restriction of $\tilde{f}$ to the straight line $G_{v_{0}}=\left\{\mu v_{0} /\left|v_{0}\right| \in \mathbb{R}^{2 \times 2} \mid \mu \in \mathbb{R}\right\}$. This function $\widetilde{h}: \mathbb{R} \rightarrow \mathbb{R}$ is defined by $\widetilde{h}(\mu)=\widetilde{f}\left(\mu v_{0} /\left|v_{0}\right|\right)$. We form the convex envelope of $\widetilde{h}$ which can be represented, by Theorem 5.6, as

$$
\widetilde{h}^{c}(\mu)=\inf \left\{\lambda \widetilde{h}\left(\mu^{\prime}\right)+(1-\lambda) \widetilde{h}\left(\mu^{\prime \prime}\right) \mid \mu=\lambda \mu^{\prime}+(1-\lambda) \mu^{\prime \prime}, 0 \leqslant \lambda \leqslant 1\right\} .
$$

The graph of $\widetilde{h} \mid[-1,1]$ is a semi-circle. Its tangent through the point $\left(\mu^{\prime \prime}, \widetilde{h}\left(\mu^{\prime \prime}\right)\right)=\left(\left|v_{0}\right|, \widetilde{f}\left(v_{0}\right)\right)$ meets the graph of $\widetilde{h} \mid[-1,1]$ in some point $\left(\mu^{\prime}, \widetilde{h}\left(\mu^{\prime}\right)\right)=\left(\mu^{\prime}, f_{0}\left(\mu^{\prime} v_{0} /\left|v_{0}\right|\right)\right)$ with $-1<\mu^{\prime}<1$ while the point $(1, \widetilde{h}(1))=\left(1, f_{0}\left(v_{0} /\left|v_{0}\right|\right)\right)$ is situated above the tangent. We choose $0<\lambda<1$ with $\lambda \mu^{\prime}+(1-\lambda) \mu^{\prime \prime}=1$ and find

$$
\widetilde{h}^{c}(1) \leqslant \lambda \widetilde{h}\left(\mu^{\prime}\right)+(1-\lambda) \widetilde{h}\left(\mu^{\prime \prime}\right)<\widetilde{h}(1) .
$$

Since the difference of arbitrary two points of $\mathrm{G}_{v_{0}}$ is a rank one matrix, it holds for all $\mu \in \mathbb{R}$ :

$$
\widetilde{f}^{c}\left(\mu v_{0} /\left|v_{0}\right|\right) \leqslant \widetilde{f}^{q c}\left(\mu v_{0} /\left|v_{0}\right|\right) \leqslant \widetilde{f}^{r c}\left(\mu v_{0} /\left|v_{0}\right|\right) \leqslant \widetilde{h}^{c}(\mu),
$$

and in particular, we deduce for $v_{0} /\left|v_{0}\right| \in \partial \mathrm{K}$ :

$$
\widetilde{f}^{c}\left(v_{0} /\left|v_{0}\right|\right) \leqslant \widetilde{f}^{q c}\left(v_{0} /\left|v_{0}\right|\right) \leqslant \widetilde{f}^{r c}\left(v_{0} /\left|v_{0}\right|\right) \leqslant \widetilde{h}^{c}(1)<\widetilde{h}(1)=f_{0}\left(v_{0} /\left|v_{0}\right|\right) .
$$

Since all functions within (2.35) are continuous on $\mathrm{K}$, the inequalities remain valid for points $v \in$ int $(\mathrm{K})$ sufficiently close to $v_{0} /\left|v_{0}\right|$.

(4) Obviously, the function $f: \mathbb{R}^{n m} \rightarrow \overline{\mathbb{R}}$ belongs to $\mathcal{F}_{K}$ and is lower semicontinuous as well as convex. Then by Lemma $2.13, f$ is a quasiconvex function.

Proof of Theorem 2.23. (5) It is obvious that $f^{c}$ admits the claimed representation. By Theorem 2.17(2), this function is lower semicontinuous and quasiconvex. 


\section{The Dacorogna-Marcellini envelope $f^{*}$ And its Relation to $f^{(q c)}$}

In the present chapter, we trace Dacorogna/Marcellini's idea to receive a "quasiconvex" envelope $f^{*}$, which is adapted to the control restriction in $(\mathrm{P})$ by introducing the restriction $v+J x(t) \in \mathrm{K}(\forall) t \in \Omega$ into the representation formula (2.10) for $f^{q c}{ }^{39}$. The following sections are devoted to a detailed study of $f^{*}$. In this study, the assertions from [14] will be extended and partly corrected. In general, one cannot prove that $f^{*}$ is a quasiconvex function ${ }^{40}$. Nevertheless, $f^{*}$ and $f^{(q c)}$ agree on int $(\mathrm{K})$, and the values of $f^{(q c)}$ on $\partial \mathrm{K}$ can be calculated by a radial limit passage. Throughout the whole section, we fix a convex body $\mathrm{K} \subset \mathbb{R}^{n m}$ with $\mathfrak{o} \in \operatorname{int}(\mathrm{K})$ and the quantities $c_{\mathrm{K}}=\operatorname{Dist}(\mathfrak{o}, \partial \mathrm{K})$ and $C_{\mathrm{K}}=\operatorname{Max}\left(1, \operatorname{Max}_{v \in \mathrm{K}}|v|\right)$, thus $0<c_{\mathrm{K}} \leqslant C_{\mathrm{K}}$ and $\operatorname{Diam}(\mathrm{K}) \leqslant 2 C_{\mathrm{K}}$.

\subsection{The envelope $f^{*}$ related to $\mathrm{K}$}

In this section, we present the definition of $f^{*}$ together with some of its basic properties.

Definition 3.1 (Envelope $f^{*}$ related to K). Let $f: \mathbb{R}^{n m} \rightarrow \overline{\mathbb{R}}$ be a function with the following properties: the set $\operatorname{dom}(f)$ is measurable, $f \mid \operatorname{dom}(f)$ is a measurable function, and $f$ is bounded from below on $\mathbb{R}^{\text {nm }}$. $\mathrm{K} \subset \mathbb{R}^{n m}$ is the above mentioned convex body. Then we define for $v \in \mathbb{R}^{n m}$ :

$$
f^{*}(v)=\inf \left\{\frac{1}{|\Omega|} \int_{\Omega} f(v+J x(t)) \mathrm{d} t \mid x \in W_{0}^{1, \infty}\left(\Omega, \mathbb{R}^{n}\right), v+J x(t) \in \mathrm{K}(\forall) t \in \Omega\right\} \in \overline{\mathbb{R}} .
$$

If the dependence on $\mathrm{K}$ should be expressed explicitly then we write also $f^{*}(\mathrm{~K})$.

Remarks. (a) The function $f^{*}$ was introduced in [25], p. 356, in the special case $\mathrm{K}=\mathrm{K}(\mathfrak{o}, \varrho)$ and in [14], p. 27, Theorem 7.2 , for arbitrary convex bodies $\mathrm{K}$. In contrast to both these papers where $f \in C^{0}(\mathrm{~K}, \mathbb{R})$ has been assumed, we formulate the definition from the outset for functions $f: \mathbb{R}^{n m} \rightarrow \overline{\mathbb{R}}$.

(b) $f^{*}$ arises as the pointwise infimum of the uncountable family $\left\{f_{x} \mid x \in W_{0}^{1, \infty}\left(\Omega, \mathbb{R}^{n}\right)\right\}$ with $f_{x}: \mathbb{R}^{n m} \rightarrow \overline{\mathbb{R}}$ defined as $v \longmapsto f_{x}(v)=\frac{1}{|\Omega|} \int_{\Omega} f(J x(t)+v) \mathrm{d} t$.

(c) The following theorem shows that the definition does not depend on the choice of $\Omega$.

Theorem $3.2\left(f^{*} \text { is defined independently of } \Omega\right)^{41}$. Let $\mathrm{K} \subset \mathbb{R}^{n m}$ and $f: \mathbb{R}^{n m} \rightarrow \overline{\mathbb{R}}$ as in Definition 3.1 be given. If both sets $\Omega, \widetilde{\Omega} \subset \mathbb{R}^{m}$ are closures of bounded strongly Lipschitz domains then

$$
\begin{aligned}
f^{*}(v) & =\inf \left\{\frac{1}{|\Omega|} \int_{\Omega} f(v+J x(t)) \mathrm{d} t \mid x \in W_{0}^{1, \infty}\left(\Omega, \mathbb{R}^{n}\right), v+J x(t) \in \mathrm{K}(\forall) t \in \Omega\right\} \\
& =\inf \left\{\frac{1}{|\widetilde{\Omega}|} \int_{\widetilde{\Omega}} f(v+J x(t)) \mathrm{d} t \mid x \in W_{0}^{1, \infty}\left(\widetilde{\Omega}, \mathbb{R}^{n}\right), v+J x(t) \in \mathrm{K}(\forall) t \in \widetilde{\Omega}\right\} .
\end{aligned}
$$

From this point, we consider functions $f \in \mathcal{F}_{\mathrm{K}}$ only. The function $f^{*}$ can be arranged with the other generalized convex envelopes of $f$ in the following way:

Theorem 3.3. Let $f \in \mathcal{F}_{\mathrm{K}}$ be given.

(1) (Inequalities between $f^{(q c)}, f^{*}$ and $f$ ) It holds:

$$
f^{(q c)}(v) \leqslant f^{*}(v) \leqslant f(v) \quad \forall v \in \mathbb{R}^{n m} .
$$

\footnotetext{
39 [14], p. 27.

${ }^{40}$ In [14], p. 27, Theorem 7.2, the validity of Morrey's integral inequality is claimed for all $v \in \operatorname{int}(\mathrm{K})$ only. Cf. Theorem 3.17(1) in Section 3.4 below.

$41_{[14], \text { p. } 28 \text { f., Step } 1 .}$
} 
(2) ( $f^{*}$ as upper bound for quasiconvex functions $\left.g \leqslant f\right)^{42}$ For any quasiconvex function $g: \mathbb{R}^{n m} \rightarrow \overline{\mathbb{R}}$, we have the implication

$$
g(v) \leqslant f(v) \quad \forall v \in \mathbb{R}^{n m} \Longrightarrow g(v) \leqslant f^{*}(v) \quad \forall v \in \mathbb{R}^{n m}
$$

Remark. Part (2) does not follow from Part (1) since the quasiconvex function $g$ must not necessarily be lower semicontinuous. From Definition 3.1 and Theorem 2.11 it follows:

Theorem 3.4 ( $f^{*}$ on the faces of $\left.\mathrm{K}\right)$. Let $f \in \mathcal{F}_{\mathrm{K}}$ and a $k$-dimensional face $\Phi$ of $\mathrm{K}, 0 \leqslant k \leqslant n m$, be given. Then it follows:

$(1)^{43}$ For all $v \in \Phi$, we have

$$
f^{*}(v)=\inf \left\{\frac{1}{|\Omega|} \int_{\Omega} f(v+J x(t)) \mathrm{d} t \mid x \in W_{0}^{1, \infty}\left(\Omega, \mathbb{R}^{n}\right), v+J x(t) \in \Phi(\forall) t \in \Omega\right\} .
$$

(2) For all $v \in \operatorname{ri}(\Phi)$, we have

$$
f^{*}(v)=\inf \left\{\frac{1}{|\Omega|} \int_{\Omega} f(v+J x(t)) \mathrm{d} t \mid x \in W_{0}^{1, \infty}\left(\Omega, \mathbb{R}^{n}\right), v+J x(t) \in \operatorname{ri}(\Phi)(\forall) t \in \Omega\right\} .
$$

(3) In particular, $f^{*}(v)=f(v)$ for all $v \in \operatorname{ext}(\mathrm{K})$.

(4) Moreover, it holds $f^{*}(v)=+\infty$ for all $v \in \mathbb{R}^{n m} \backslash \mathrm{K}$.

Proof of Theorem 3.3. (1) From the proof of Theorem 2.11(2) we see that for $v \in \mathbb{R}^{n m} \backslash \mathrm{K}$, the infimum in Definition 3.1 is formed over an empty set, thus taking the value $+\infty$. By Theorem 2.18(1), the claimed inequality is valid in these points. Given now $v \in \mathrm{K}$ and a function $x \in W_{0}^{1 \infty}\left(\Omega, \mathbb{R}^{n}\right)$ with $v+J x(t) \in \mathrm{K}$ $(\forall) t \in \Omega$, it holds that

$$
f^{(q c)}(v+J x(t)) \leqslant f(v+J x(t)) \quad(\forall) t \in \Omega \Longrightarrow \frac{1}{|\Omega|} \int_{\Omega} f^{(q c)}(v+J x(t)) \mathrm{d} t \leqslant \frac{1}{|\Omega|} \int_{\Omega} f(v+J x(t)) \mathrm{d} t .
$$

With Theorem 2.11(2), we derive from the quasiconvexity of $f^{(q c)}$ (Thm. 2.18(2)):

$$
\begin{aligned}
f^{(q c)}(v) & =\inf \left\{\frac{1}{|\Omega|} \int_{\Omega} f^{(q c)}(v+J x(t)) \mathrm{d} t \mid x \in W_{0}^{1, \infty}\left(\Omega, \mathbb{R}^{n}\right), v+J x(t) \in \mathrm{K}(\forall) t \in \Omega\right\} \\
& \leqslant \inf \left\{\frac{1}{|\Omega|} \int_{\Omega} f(v+J x(t)) \mathrm{d} t \mid x \in W_{0}^{1, \infty}\left(\Omega, \mathbb{R}^{n}\right), v+J x(t) \in \mathrm{K}(\forall) t \in \Omega\right\}=f^{*}(v) .
\end{aligned}
$$

The inequality $f^{*}(v) \leqslant f(v)$ results from the feasibility of $x=\mathfrak{o}$ in the forming of the infimum for all $v \in \mathrm{K}$.

Proof of Theorem 3.4. (2) Choose $v \in \operatorname{ri}(\Phi)$. By Part (1), for every $\varepsilon>0$ there exists some $x \in W_{0}^{1, \infty}\left(\Omega, \mathbb{R}^{n}\right)$ with

$$
f^{*}(v) \leqslant \frac{1}{|\Omega|} \int_{\Omega} f(v+J x(t)) \mathrm{d} t \leqslant f^{*}(v)+\varepsilon \text { and } v+J x(t) \in \Phi(\forall) t \in \Omega .
$$

Describe the uniform continuity of $f$ on $\mathrm{K} \cap \Phi$ through the $\varepsilon-\delta$ relation

$$
\left|v^{\prime}-v^{\prime \prime}\right| \leqslant \delta(\varepsilon) \Longrightarrow\left|f\left(v^{\prime}\right)-f\left(v^{\prime \prime}\right)\right| \leqslant \varepsilon \quad \forall v^{\prime}, v^{\prime \prime} \in \Phi .
$$

\footnotetext{
42 [41], p. 240, Theorem 4, i).

${ }^{43}$ Parts 1), 3) and 4): [41], p. 239 f., Theorem 3.
} 
Then there exists a number $0<\lambda<1$ with

$$
\begin{aligned}
|(v+J x(t))-(v+\lambda J x(t))|=(1-\lambda)|J x(t)| \leqslant & \delta(\varepsilon)(\forall) t \in \Omega \Longrightarrow \\
& |f(v+J x(t))-f(v+\lambda J x(t))| \leqslant \varepsilon \quad(\forall) t \in \Omega,
\end{aligned}
$$

since $v \in \operatorname{ri}(\Phi)$ implies $v+\lambda J x(t) \in \operatorname{ri}(\Phi)(\forall) t \in \Omega$. We further conclude that

$$
\begin{aligned}
& \frac{1}{|\Omega|} \int_{\Omega}(f(v+J x(t))-f(v+\lambda J x(t))) \mathrm{d} t+\frac{1}{|\Omega|} \int_{\Omega} f(v+\lambda J x(t)) \mathrm{d} t \leqslant f^{*}(v)+\varepsilon \Longrightarrow \\
& -\frac{1}{|\Omega|} \int_{\Omega}|f(v+J x(t))-f(v+\lambda J x(t))| \mathrm{d} t+\frac{1}{|\Omega|} \int_{\Omega} f(v+\lambda J x(t)) \mathrm{d} t \leqslant f^{*}(v)+\varepsilon \Longrightarrow \\
& -\varepsilon+f^{*}(v) \leqslant-\varepsilon+\frac{1}{|\Omega|} \int_{\Omega} f(v+\lambda J x(t)) \mathrm{d} t \leqslant f^{*}(v)+\varepsilon,
\end{aligned}
$$

and the infimum will not be changed if $x \in W_{0}^{1, \infty}\left(\Omega, \mathbb{R}^{n}\right)$ varies over functions with $v+J x(t) \in \operatorname{ri}(\Phi)(\forall) t \in \Omega$ only.

\subsection{Continuity, semicontinuity and measurability of $f^{*}$}

We are interested now in the analytical properties of the envelope $f^{*}$. The most important result is expressed in the following theorem: $f^{*}$ obeys a $\varepsilon-\delta$ continuity relation depending not only on the distance of the given points $v^{\prime}, v^{\prime \prime} \in \Phi$ but also on their distances to the relative boundary $\operatorname{rb}(\Phi)$ of the face $\Phi \subset \mathrm{K}$.

Theorem 3.5 ( $\varepsilon-\delta$ relation for the restriction of $f^{*}$ to faces of $\left.\mathrm{K}\right)$. Let $f \in \mathcal{F}_{\mathrm{K}}$ and a $k$-dimensional face $\Phi \subseteq \mathrm{K}, 0 \leqslant k \leqslant n m$, be given. Assume that the uniform continuity of $f$ on $\mathrm{K}$ is described through the $\varepsilon-\delta$ relation

$$
\left|v^{\prime}-v^{\prime \prime}\right| \leqslant \delta(\varepsilon)<1 \Longrightarrow\left|f\left(v^{\prime}\right)-f\left(v^{\prime \prime}\right)\right| \leqslant \varepsilon \quad \forall v^{\prime}, v^{\prime \prime} \in \mathrm{K} .
$$

Then $f^{*} \mid \Phi$ obeys the following $\varepsilon-\delta$ relation:

$$
\begin{aligned}
&\left|v^{\prime}-v^{\prime \prime}\right| \leqslant \delta_{1}(\varepsilon) \cdot \operatorname{Min}\left(1, \operatorname{Dist}\left(v^{\prime}, \operatorname{rb}(\Phi)\right), \operatorname{Dist}\left(v^{\prime \prime}, \operatorname{rb}(\Phi)\right)\right) \Longrightarrow \Longrightarrow \\
&\left|f^{*}\left(v^{\prime}\right)-f^{*}\left(v^{\prime \prime}\right)\right| \leqslant 2 \varepsilon \quad \forall v^{\prime}, v^{\prime \prime} \in \operatorname{ri}(\Phi)
\end{aligned}
$$

with $\delta_{1}(\varepsilon)=\frac{1}{4} \delta(\varepsilon) / C_{\mathrm{K}}$ where $C_{\mathrm{K}}$ is the quantity defined in the beginning of the section.

Due to this theorem, all restrictions $f^{*} \mid \operatorname{ri}(\Phi)$ are continuous while the upper semicontinuity and even measurability of $f^{*} \mid \mathrm{K}$ itself can be proven under special assumptions about the facial structure of $\mathrm{K}$ only. In detail, we will prove the following assertions:

Theorem 3.6 (Continuity of $f^{*}$ ). Let $f \in \mathcal{F}_{\mathrm{K}}$ and a $k$-dimensional face $\Phi \subseteq \mathrm{K}, 0 \leqslant k \leqslant n m$, be given.

$(1)^{44}$ The restriction $f^{*} \mid \Phi$ is continuous in every point $v \in \mathrm{ri}(\Phi)$. In particular, $f^{*}$ is continuous in every point $v \in \operatorname{int}(\mathrm{K})$.

(2) $f^{*} \mid \mathrm{K}$ is continuous in every point $v \in \operatorname{ext}(\mathrm{K})$.

(3) If $\partial \mathrm{K}=\operatorname{ext}(\mathrm{K})$ then $f^{*} \mid \mathrm{K}$ is continuous on the whole set $\mathrm{K}$, and $f^{*}$ belongs to $\mathcal{F}_{\mathrm{K}}$.

Theorem 3.7 (Upper limit of the restriction of $f^{*}$ to faces of $\left.\mathrm{K}\right)^{45}$. Let $f \in \mathcal{F}_{\mathrm{K}}$ and a k-dimensional face $\Phi \subseteq \mathrm{K}, 0<k \leqslant n m$, be given. Then along any sequence of points $\left\{v^{N}\right\}$, ri $(\Phi) \rightarrow v_{0} \in \operatorname{rb}(\Phi)$, we have $\limsup _{N \rightarrow \infty} f^{*}\left(v^{N}\right) \leqslant f^{*}\left(v_{0}\right)$.

\footnotetext{
${ }^{44}$ Generalization of [14], p. 29, (7.17).

${ }^{45}$ Generalization of [14], p. 29, (7.18).
} 


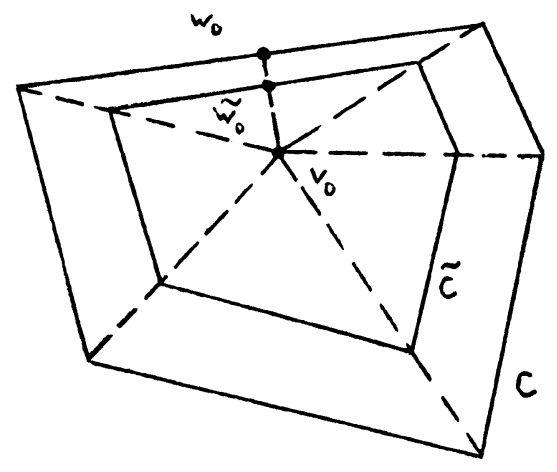

FiguRE 1.

Theorem 3.8 (Upper semicontinuity of $f^{*}$ ). Let $f \in \mathcal{F}_{\mathrm{K}}$ be given.

(1) If $\mathrm{K}$ is a polytope then $f^{*} \mid \mathrm{K}$ is upper semicontinuous on $\mathrm{K}$.

(2) If $\mathrm{K}$ is, more generally, a convex body with only finitely many faces of dimensions $1 \leqslant k<n m$ then $f^{*} \mid \mathrm{K}$ is upper semicontinuous on $\mathrm{K}$.

In the case of an even more general facial structure of K, however, the upper semicontinuity of $f^{*}$ cannot be derived from Theorem $3.7^{46}$. It results from Theorem 3.6(1) that for $f \in \mathcal{F}_{K}, f^{*}$ is a $\left(\mathbb{R}^{n m}, \mathfrak{L}^{n m}\right)-\left(\mathbb{R}, \mathfrak{B}^{1}\right)$ measurable function since the inverse image of a open set $\mathrm{A} \subseteq \mathbb{R}$ under $f^{*}$ can be represented as union $\left\{v \in \mathbb{R}^{n m} \mid f^{*}(v) \in \mathrm{A}\right\}=\left\{v \in \operatorname{int}(\mathrm{K}) \mid f^{*}(v) \in \mathrm{A}\right\} \cup\left\{v \in \partial \mathrm{K} \mid f^{*}(v) \in \mathrm{A}\right\}$ of a Borel set and a subset of the $n m$-dimensional Lebesgue null set $\partial \mathrm{K}$ (Lem. 5.3). About the Borel measurability of $f^{*}$, however, only the following proposition can be confirmed.

Theorem 3.9 (Borel measurability of $f^{*}$ ). Let $f \in \mathcal{F}_{\mathrm{K}}$ be given. If $\mathrm{K}$ possesses at most countably many faces $\Phi_{s}$ of dimension $1 \leqslant k \leqslant n m$ then $f^{*} \mid \mathrm{K}$ belongs to the second Baire class and is, consequently, Borel measurable.

Proof of Theorem 3.5 .

- Step 1. An inequality for compact, convex sets. The proof of Theorem 3.5 is based on the following geometrical proposition:

Lemma 3.10. Let a nonempty, convex, compact set $\mathrm{C} \subset \mathbb{R}^{n m}$ with $v_{0} \in \operatorname{ri}(\mathrm{C})$ and a function $x \in W_{0}^{1, \infty}\left(\Omega, \mathbb{R}^{n}\right)$ be given. Then it holds for all $0<\lambda<1$ :

$$
v_{0}+J x(t) \in \operatorname{ri}(\mathrm{C})(\forall) t \in \Omega \Longrightarrow \lambda \cdot \operatorname{Dist}\left(v_{0}, \operatorname{rb}(\mathrm{C})\right) \leqslant \operatorname{Dist}\left(v_{0}+(1-\lambda) J x(t), \operatorname{rb}(\mathrm{C})\right)(\forall) t \in \Omega .
$$

Proof. At first, we prove the inequality in the case that $\mathrm{C}$ is a $k$-dimensional polytope. Denote by $\widetilde{\mathrm{C}}=\mathcal{S}(\mathrm{C})$ the image of $\mathrm{C}$ under the homothety $\mathcal{S}$ with center $v_{0}$ and ratio $(1-\lambda)$, i.e. $\mathcal{S}(v)=v_{0}+(1-\lambda)\left(v-v_{0}\right)$. Since $\mathrm{C}$ is a polytope, the distances $\operatorname{Dist}\left(v_{0}, \operatorname{rb}(\mathrm{C})\right)=\operatorname{Dist}\left(v_{0}, w_{0}\right)$ and $\operatorname{Dist}\left(v_{0}, \operatorname{rb}(\widetilde{\mathrm{C}})\right)=\operatorname{Dist}\left(v_{0}, \widetilde{w}_{0}\right)$ will be taken on in points $w_{0} \in \operatorname{rb}(\mathrm{C}) \operatorname{resp} . \widetilde{w}_{0}=\mathcal{S}\left(w_{0}\right) \in \operatorname{rb}(\widetilde{\mathrm{C}})$, and at the same time, we find $\operatorname{Dist}(\operatorname{rb}(\mathrm{C}), \operatorname{rb}(\widetilde{\mathrm{C}}))=$ $\left|w_{0}-\widetilde{w}_{0}\right|=\lambda \cdot\left|w_{0}-v_{0}\right|=\lambda \cdot \operatorname{Dist}\left(v_{0}, \operatorname{rb}(\mathrm{C})\right)\left(c f\right.$. Fig. 1). For almost all $t \in \Omega$ it follows: $v_{0}+J x(t) \in \mathrm{C} \Longrightarrow$ $v_{0}+(1-\lambda) J x(t) \in \widetilde{\mathrm{C}} \cap \operatorname{ri}(\mathrm{C}) \Longrightarrow$

$$
\operatorname{Dist}\left(v_{0}+(1-\lambda) J x(t), \operatorname{rb}(\mathrm{C})\right) \geqslant \operatorname{Dist}(\widetilde{\mathrm{C}}, \mathrm{rb}(\mathrm{C}))=\operatorname{Dist}(\operatorname{rb}(\widetilde{\mathrm{C}}), \mathrm{rb}(\mathrm{C}))=\lambda \cdot \operatorname{Dist}\left(v_{0}, \mathrm{rb}(\mathrm{C})\right) .
$$

\footnotetext{
${ }^{46}$ The according derivation in [14], p. 31, third line from the bottom, is erroneous. However, instead of the assertion ibid., (7.29), we prove Theorem 3.17. below.
} 
By Theorem 5.5(1), any nonempty, convex, compact set $\mathrm{C}$ can be approximated with convex polytopes with respect to the convergence in Hausdorff distance: For every $0<\varepsilon<\operatorname{Dist}\left(v_{0}, \mathrm{rb}(\mathrm{C})\right)$, one can find polytopes $\mathrm{P}_{\varepsilon}^{\prime}$ and $\mathrm{P}_{\varepsilon}^{\prime \prime}=\left(\mathrm{P}_{\varepsilon}^{\prime}+\mathrm{Q}(\mathfrak{o}, \varepsilon)\right)$ with

$$
\mathrm{P}_{\varepsilon}^{\prime} \subseteq \mathrm{C} \subseteq \mathrm{P}_{\varepsilon}^{\prime \prime}=\left(\mathrm{P}_{\varepsilon}^{\prime}+\mathrm{Q}(\mathfrak{o}, \varepsilon)\right) \subset\left(\mathrm{P}_{\varepsilon}^{\prime}+\mathrm{K}(\mathfrak{o}, \varepsilon)\right)
$$

where $\mathrm{Q}(\mathfrak{o}, \varepsilon)$ denotes the closed cube with center $\mathfrak{o}$ and edge length $\varepsilon$. Thus we have for all points $w \in \mathrm{C}$ :

$$
0 \leqslant \operatorname{Dist}\left(w \operatorname{rb}\left(\mathrm{P}_{\varepsilon}^{\prime \prime}\right)\right)-\operatorname{Dist}(w, \operatorname{rb}(\mathrm{C})) \text { and } \operatorname{Dist}\left(w, \operatorname{rb}\left(\mathrm{P}_{\varepsilon}^{\prime \prime}\right)\right)-\operatorname{Dist}(w, \operatorname{rb}(\mathrm{C})) \leqslant \varepsilon .
$$

The inequality (3.19) can be applied to the polytope $\mathrm{P}_{\varepsilon}^{\prime \prime}$ :

$$
\lambda \cdot \operatorname{Dist}\left(v_{0}, \operatorname{rb}\left(\mathrm{P}_{\varepsilon}^{\prime \prime}\right)\right) \leqslant \operatorname{Dist}\left(v_{0}+(1-\lambda) J x(t), \operatorname{rb}\left(\mathrm{P}_{\varepsilon}^{\prime \prime}\right)\right) .
$$

It follows that

$$
\begin{array}{r}
\lambda \cdot \operatorname{Dist}\left(v_{0}, \mathrm{rb}(\mathrm{C})\right)-\lambda \cdot\left|\operatorname{Dist}\left(v_{0}, \mathrm{rb}\left(\mathrm{P}_{\varepsilon}^{\prime \prime}\right)\right)-\operatorname{Dist}\left(v_{0}, \mathrm{rb}(\mathrm{C})\right)\right| \leqslant \operatorname{Dist}\left(v_{0}+(1-\lambda) J x(t), \mathrm{rb}(\mathrm{C})\right) \\
+\left|\operatorname{Dist}\left(v_{0}+(1-\lambda) J x(t), \mathrm{rb}\left(\mathrm{P}_{\varepsilon}^{\prime \prime}\right)\right)-\operatorname{Dist}\left(v_{0}+(1-\lambda) J x(t), \mathrm{rb}(\mathrm{C})\right)\right| \Longrightarrow \\
\lambda \cdot \operatorname{Dist}\left(v_{0}, \mathrm{rb}(\mathrm{C})\right) \leqslant \operatorname{Dist}\left(v_{0}+(1-\lambda) J x(t), \mathrm{rb}(\mathrm{C})\right)+2 \varepsilon
\end{array}
$$

and by the limit passage $\varepsilon \rightarrow 0$, we arrive at the claimed assertion.

- Step 2. Derivation of the $\varepsilon-\delta$ relation. $f$ is uniformly continuous on $\mathrm{K}$ as well as on $\mathrm{K} \cap \Phi$ with the $\varepsilon-\delta$ relation

$$
\left|v^{\prime}-v^{\prime \prime}\right| \leqslant \delta(\varepsilon)<1 \Longrightarrow\left|f\left(v^{\prime}\right)-f\left(v^{\prime \prime}\right)\right| \leqslant \varepsilon \quad \forall v^{\prime}, v^{\prime \prime} \in \Phi .
$$

Let us fix now $v \in \operatorname{ri}(\Phi)$. By Theorem 3.4(2) there exists a function $x \in W_{0}^{1, \infty}\left(\Omega, \mathbb{R}^{n}\right)$ with

$$
f^{*}(v) \leqslant \frac{1}{|\Omega|} \int_{\Omega} f(v+J x(t)) \mathrm{d} t \leqslant f^{*}(v)+\varepsilon \text { and } v+J x(t) \in \operatorname{ri}(\Phi)(\forall) t \in \Omega .
$$

From $|v| \leqslant C_{\mathrm{K}}$ and $|v+J x(t)| \leqslant C_{\mathrm{K}}(\forall) t \in \Omega$, it follows that $|J x(t)| \leqslant 2 C_{\mathrm{K}}(\forall) t \in \Omega$. Defining $\lambda=$ $\frac{1}{4} \delta(\varepsilon) / C_{\mathrm{K}}$, the points $v+(1-\lambda) J x(t)=\lambda v+(1-\lambda)(v+J x(t))$ as well belong to the convex set ri $(\Phi)$ $(\forall) t \in \Omega$. By Lemma 3.10 we have for all $w \in \operatorname{ri}(\Phi)$ the implication

$$
\begin{aligned}
|v-w| \leqslant \lambda \cdot \operatorname{Dist}(v, \operatorname{rb}(\Phi)) & \leqslant \operatorname{Dist}(v+(1-\lambda) J x(t), \operatorname{rb}(\Phi)) \Longrightarrow \\
w+(1-\lambda) J x(t) & =(w-v)+(v+(1-\lambda) J x(t)) \in \Phi
\end{aligned}
$$

Now let $\delta_{2}(\varepsilon, v)=\operatorname{Min}\left(\frac{1}{2} \delta(\varepsilon), \lambda \cdot \operatorname{Dist}(v, \operatorname{rb}(\Phi))\right)$. Then for all $w \in \operatorname{ri}(\Phi)$ it follows that

$$
\begin{aligned}
& |v-w| \leqslant \delta_{2}(\varepsilon, v) \Longrightarrow|(v+J x(t))-(w+(1-\lambda) J x(t))| \leqslant|v-w|+\lambda \cdot|J x(t)| \\
\leqslant & \frac{\delta(\varepsilon)}{2}+\frac{\delta(\varepsilon)}{4 C_{\mathrm{K}}} \cdot 2 C_{\mathrm{K}}=\delta(\varepsilon) \Longrightarrow|f(v+J x(t))-f(w+(1-\lambda) J x(t))| \leqslant \varepsilon \quad(\forall) t \in \Omega .
\end{aligned}
$$

Consequently, we have for all $w \in \operatorname{ri}(\Phi)$ :

$$
\begin{aligned}
& \quad|v-w| \leqslant \delta_{2}(\varepsilon, v) \Longrightarrow \\
& \frac{1}{|\Omega|} \int_{\Omega}(f(v+J x(t))-f(w+(1-\lambda) J x(t))) \mathrm{d} t+\frac{1}{|\Omega|} \int_{\Omega} f(w+(1-\lambda) J x(t)) \mathrm{d} t \leqslant f^{*}(v)+\varepsilon \Longrightarrow \\
& \quad-\varepsilon+f^{*}(w) \leqslant-\varepsilon+\frac{1}{|\Omega|} \int_{\Omega} f(w+(1-\lambda) J x(t)) \mathrm{d} t \leqslant f^{*}(v)+\varepsilon \Longrightarrow f^{*}(w)-f^{*}(v) \leqslant 2 \varepsilon
\end{aligned}
$$


After exchanging the roles of $v$ and $w$, we find, conversely, for all $v \in \operatorname{ri}(\Phi)$ :

$$
|w-v| \leqslant \delta_{2}(\varepsilon, w)=\operatorname{Min}\left(\frac{\delta(\varepsilon)}{2}, \lambda \cdot \operatorname{Dist}(w, \operatorname{rb}(\Phi))\right) \Longrightarrow f^{*}(v)-f^{*}(w) \leqslant 2 \varepsilon
$$

Summing up, for all $v, w \in \operatorname{ri}(\Phi)$ it holds that

$$
\begin{array}{r}
|v-w| \leqslant \operatorname{Min}\left(\delta_{2}(\varepsilon, v), \delta_{2}(\varepsilon, w)\right)=\operatorname{Min}\left(\frac{\delta(\varepsilon)}{2}, \frac{\delta(\varepsilon)}{4 C_{\mathrm{K}}} \cdot \operatorname{Dist}(v, \operatorname{rb}(\Phi)), \frac{\delta(\varepsilon)}{4 C_{\mathrm{K}}} \cdot \operatorname{Dist}(w, \operatorname{rb}(\Phi))\right) \\
\Longrightarrow\left|f^{*}(v)-f^{*}(w)\right| \leqslant 2 \varepsilon
\end{array}
$$

from which the assertion of the theorem follows while $\delta_{1}(\varepsilon)=\frac{1}{4} \delta(\varepsilon) / C_{\mathrm{K}}$.

Proof of Theorem 3.6. (1) Let $v \in \operatorname{ri}(\Phi)$ and $\varepsilon>0$ be given. We choose a number $0<\varrho<1$ such that at the same time

$$
(\mathrm{K}(v, \varrho) \cap \Phi) \subset \operatorname{ri}(\Phi) \text { and } \varrho \leqslant \operatorname{Dist}((\mathrm{K}(v, \varrho) \cap \Phi), \operatorname{rb}(\Phi))
$$

hold. It follows that

$$
\varrho \leqslant \operatorname{Min}(1, \operatorname{Dist}((\mathrm{K}(v, \varrho) \cap \Phi), \operatorname{rb}(\Phi)))=\underset{z \in \mathrm{K}(v, \varrho) \cap \Phi}{\operatorname{Min}} \operatorname{Min}(1, \operatorname{Dist}(v, \operatorname{rb}(\Phi)), \operatorname{Dist}(z, \operatorname{rb}(\Phi)))
$$

and by Theorem 3.5, we get for all $w \in\left(\mathrm{K}\left(v, \delta_{1}(\varepsilon) \cdot \varrho\right) \cap \Phi\right) \subset(\mathrm{K}(v, \varrho) \cap \Phi) \subset \operatorname{ri}(\Phi)$ :

$$
\begin{aligned}
|v-w| & \leqslant \delta_{1}(\varepsilon) \cdot \varrho \leqslant \delta_{1}(\varepsilon) \cdot \underset{z \in \mathrm{K}(v, \varrho) \cap \Phi}{\operatorname{Min}} \operatorname{Min}(1, \operatorname{Dist}(v, \operatorname{rb}(\Phi)), \operatorname{Dist}(z, \operatorname{rb}(\Phi))) \\
\leqslant & \delta_{1}(\varepsilon) \cdot \operatorname{Min}(1, \operatorname{Dist}(v, \operatorname{rb}(\Phi)), \operatorname{Dist}(w, \operatorname{rb}(\Phi))) \Longrightarrow\left|f^{*}(v)-f^{*}(w)\right| \leqslant 2 \varepsilon .
\end{aligned}
$$

Consequently, $f^{*} \mid \operatorname{ri}(\Phi)$ is continuous in $v \in \operatorname{ri}(\Phi)$. The assertion holds, in particular, for $\Phi=\mathrm{K}$ and $\mathrm{ri}(\mathrm{K})=$ $\operatorname{int}(\mathrm{K})$.

(2) At first, let us confirm the continuity of $f^{c} \mid \mathrm{K}$ in the point $v_{0} \in \operatorname{ext}(\mathrm{K})$. By Theorem 5.7(1) and (5), it holds: $f\left(v_{0}\right)=f^{c}\left(v_{0}\right) \leqslant \liminf _{v \rightarrow v_{0}} f^{c}(v) \leqslant \limsup _{v \rightarrow v_{0}} f^{c}(v) \leqslant \limsup _{v \rightarrow v_{0}} f(v)=f\left(v_{0}\right)$ since $f$ is continuous in $v_{0}$. Thus we have $\liminf _{v \rightarrow v_{0}} f^{c}(v)=\limsup _{v \rightarrow v_{0}} f^{c}(v)$. Consider now a sequence of points $\left\{v^{N}\right\}, \mathrm{K} \rightarrow v_{0} \in \operatorname{ext}(\mathrm{K})$. Then from Theorems 2.18(1) and 3.3(1) it follows: $f^{c}\left(v^{N}\right) \leqslant f^{*}\left(v^{N}\right) \leqslant f\left(v^{N}\right)$ for all $N \in \mathbb{N}$. By Theorem 5.7(5), $f$ and $f^{c}$ agree in $v_{0} \in \operatorname{ext}(\mathrm{K})$, and from the continuity of $f^{c} \mid \mathrm{K}$ in $v_{0}$ we find

$$
f\left(v_{0}\right)=f^{c}\left(v_{0}\right)=\lim _{N \rightarrow \infty} f^{c}\left(v^{N}\right) \leqslant \liminf _{N \rightarrow \infty} f^{*}\left(v^{N}\right) \leqslant \limsup _{N \rightarrow \infty} f^{*}\left(v^{N}\right) \leqslant \lim _{N \rightarrow \infty} f\left(v^{N}\right)=f\left(v_{0}\right) .
$$

(3) The assertion results from Parts (1) and (2).

Proof of Theorem 3.7 ${ }^{47}$. Choose a number $\varepsilon>0$ and a sequence of points $\left\{v^{N}\right\}$, ri $(\Phi) \rightarrow v_{0} \in \operatorname{rb}(\Phi)$. For $v_{0} \in \operatorname{ext}(\mathrm{K})$, the assertion already holds by Theorem 3.6(2). For that reason, let us consider a face $\Phi^{\prime} \subseteq \mathrm{rb}(\Phi)$ of $\mathrm{K}$ with $\operatorname{Dim}\left(\Phi^{\prime}\right)=k^{\prime}, 1 \leqslant k^{\prime}<k$ and $v_{0} \in \operatorname{ri}\left(\Phi^{\prime}\right)$. We describe the uniform continuity of $f$ on $\mathrm{K}$ (and thus on $\Phi$ as well) by the $\varepsilon-\delta$ relation

$$
\left|v^{\prime}-v^{\prime \prime}\right| \leqslant \delta(\varepsilon)<1 \Longrightarrow\left|f\left(v^{\prime}\right)-f\left(v^{\prime \prime}\right)\right| \leqslant \varepsilon \quad \forall v^{\prime}, v^{\prime \prime} \in \Phi
$$

\footnotetext{
${ }^{47}$ With this proof, we correct the error in [14], p. 30, third line from the bottom until last line.
} 


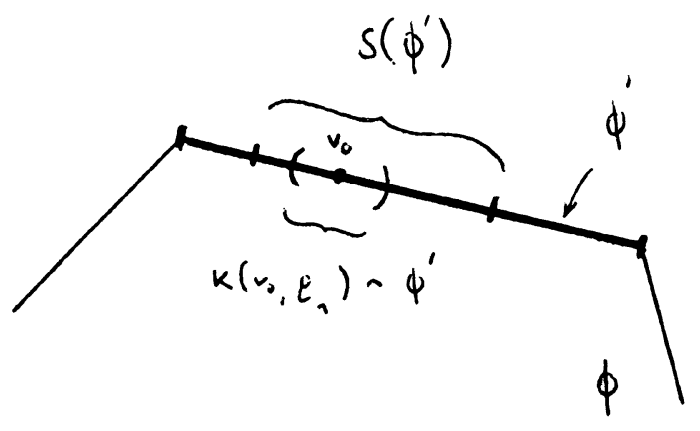

FiguRE 2.

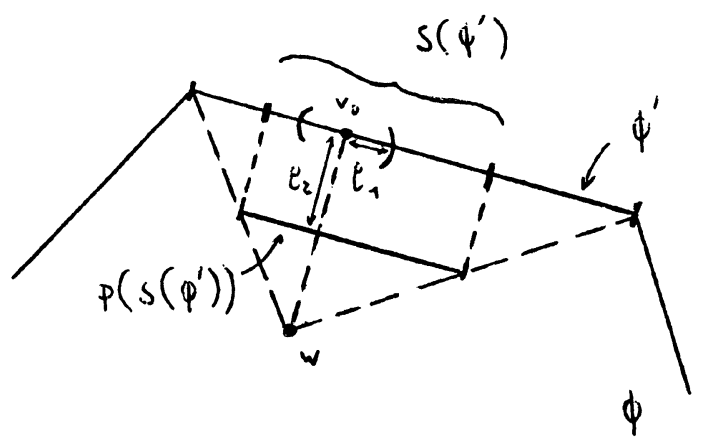

FIGURE 3.

Then, by Theorem 3.4(2), there exists some $x \in W_{0}^{1, \infty}\left(\Omega, \mathbb{R}^{n}\right)$ with

$$
f^{*}\left(v_{0}\right) \leqslant \frac{1}{|\Omega|} \int_{\Omega} f\left(v_{0}+J x(t)\right) \mathrm{d} t \leqslant f^{*}\left(v_{0}\right)+\varepsilon \text { and } v+J x(t) \in \operatorname{ri}\left(\Phi^{\prime}\right)(\forall) t \in \Omega .
$$

As in the proof of Theorem 3.5, it follows $|J x(t)| \leqslant 2 C_{\mathrm{K}}(\forall) t \in \Omega$. Defining $\lambda=\frac{1}{2} \delta(\varepsilon) / C_{\mathrm{K}}$, the points $v_{0}+(1-\lambda) J x(t)=\lambda v_{0}+(1-\lambda)\left(v_{0}+J x(t)\right)$ belong to the convex set $\Phi^{\prime}(\forall) t \in \Omega$, and we get

$$
\begin{aligned}
\left|\left(v_{0}+J x(t)\right)-\left(v_{0}+(1-\lambda) J x(t)\right)\right|= & \frac{\delta(\varepsilon)}{2 C_{\mathrm{K}}} \cdot|J x(t)| \leqslant \delta(\varepsilon) \Longrightarrow \\
& \left|f\left(v_{0}+J x(t)\right)-f\left(v_{0}+(1-\lambda) J x(t)\right)\right| \leqslant \varepsilon \quad(\forall) t \in \Omega .
\end{aligned}
$$

Denote by $\mathcal{S}$ the homothety with center $v_{0}$ and ratio $\left(1-\frac{1}{2} \lambda\right)$. First of all, then there exists a number $0<\varrho_{1}<1$ with $v+(1-\lambda) J x(t) \in \mathcal{S}\left(\Phi^{\prime}\right)(\forall) t \in \Omega$ for all $v \in \mathrm{K}\left(v_{0}, \varrho_{1}\right) \cap \Phi^{\prime}$. Furthermore, there exists a point $w \in \operatorname{ri}(\Phi)$ with $\left(w-v_{0}\right) \perp \operatorname{aff}\left(\Phi^{\prime}\right)$ since either $\Phi^{\prime}$ itself is exposed with respect to $\Phi$ or there exists a face $\widetilde{\Phi}$ with $\Phi^{\prime} \subset \widetilde{\Phi} \subset \Phi$ which is exposed with respect to $\Phi$ (cf. Fig. 2).

Let us then perform a translation $\mathcal{P}$ of $\mathcal{S}\left(\Phi^{\prime}\right)$ in direction of $\left(w-v_{0}\right)$ by the distance $0<\varrho_{2}<1$ such that $\mathcal{P}\left(\mathcal{S}\left(\Phi^{\prime}\right)\right) \subset \operatorname{co}\left(\{w\} \cup \Phi^{\prime}\right) \subseteq \Phi$ holds. Choose now $\varrho=\operatorname{Min}\left(\varrho_{1}, \varrho_{2}, \delta(\varepsilon)\right)$ (cf. Fig. 3). Then for all $v \in \mathrm{K}\left(v_{0}, \varrho\right) \cap \Phi$ it holds $v+(1-\lambda) J x(t) \in \Phi$, thus $(1-\lambda) x$ is feasible in the forming of the infimum in the definition of $f^{*}(v)$, as well as

$$
\left|\left(v_{0}+(1-\lambda) J x(t)\right)-(v+(1-\lambda) J x(t))\right| \leqslant \varrho \leqslant \delta(\varepsilon) .
$$


Consequently, we arrive at

$$
\left|f\left(v_{0}+(1-\lambda) J x(t)\right)-f(v+(1-\lambda) J x(t))\right| \leqslant \varepsilon
$$

Since all points $v^{N}$, starting from a sufficiently large index $N(\varepsilon)$, must belong to $\mathrm{K}\left(v_{0}, \varrho\right)$, it holds that

$$
v^{N}+(1-\lambda) J x(t) \in \Phi \quad \forall N \geqslant N(\varepsilon)
$$

and, consequently,

$$
\begin{aligned}
f^{*}\left(v_{0}\right)+\varepsilon \geqslant & \frac{1}{|\Omega|} \int_{\Omega}\left(f\left(v_{0}+J x(t)\right)-f\left(v_{0}+(1-\lambda) J x(t)\right)\right) \mathrm{d} t \\
& +\frac{1}{|\Omega|} \int_{\Omega}\left(f\left(v_{0}+(1-\lambda) J x(t)\right)-f\left(v^{N}+(1-\lambda) J x(t)\right)\right) \mathrm{d} t+\frac{1}{|\Omega|} \int_{\Omega} f\left(v^{N}+(1-\lambda) J x(t)\right) \mathrm{d} t \\
\geqslant & -\frac{1}{|\Omega|} \int_{\Omega}|\ldots| \mathrm{d} t-\frac{1}{|\Omega|} \int_{\Omega}|\ldots| \mathrm{d} t+\frac{1}{|\Omega|} \int_{\Omega} f\left(v^{N}+(1-\lambda) J x(t)\right) \mathrm{d} t \geqslant-2 \varepsilon+f^{*}\left(v^{N}\right)
\end{aligned}
$$

and we get $\lim \sup _{N \rightarrow \infty} f^{*}\left(v^{N}\right) \leqslant f^{*}\left(v_{0}\right)+3 \varepsilon$. Since $\varepsilon>0$ was arbitrary, the proof is complete.

Proof of Theorem 3.8. (1) is a special case of Part (2).

(2) Let us consider, accordingly, a convex body $\mathrm{K} \subset \mathbb{R}^{n m}$ with finitely many faces $\Phi_{1}, \ldots, \Phi_{s}$ of dimensions $1 \leqslant$ $k<n m$. Given a sequence of points $\left\{v^{N}\right\}, \mathrm{K} \rightarrow v_{0} \in \mathrm{K}$, a face $\Phi$ of $\mathrm{K}$ with $v_{0} \in \operatorname{ri}(\Phi)$ is uniquely determined by Lemma 5.2. If $v_{0} \in \operatorname{int}(\mathrm{K})$ or $v_{0} \in \operatorname{ext}(\mathrm{K})$ then it follows that $\limsup _{N \rightarrow \infty} f^{*}\left(v^{N}\right)=\lim _{N \rightarrow \infty} f^{*}\left(v^{N}\right)=$ $f^{*}\left(v_{0}\right)$ by Theorem 3.6(1) resp. (2). It remains to examine the case if $v_{0}$ belongs to the relative interior of one of the faces $\Phi_{1}, \ldots, \Phi_{s}$. Decompose the sequence $\left\{v^{N}\right\}$ into the finitely many (finite or infinite) subsequences $\left\{v^{N}\right\}_{N \in J_{0}},\left\{v^{N}\right\}_{N \in J_{1}}, \ldots,\left\{v^{N}\right\}_{N \in J_{s}}$ and $\left\{v^{N}\right\}_{N \in J_{s+1}}$ with $N \in J_{0} \Longleftrightarrow v^{N} \in \operatorname{ext}(\mathrm{K})$, $N \in J_{1} \Longleftrightarrow v^{N} \in \operatorname{ri}\left(\Phi_{1}\right), \ldots, N \in J_{s} \Longleftrightarrow v^{N} \in \operatorname{ri}\left(\Phi_{s}\right), N \in J_{s+1} \Longleftrightarrow v^{N} \in \operatorname{int}(\mathrm{K})$. When forming the upper limit, one has to consider the infinite subsequences only, which have to converge to $v_{0}$ again. For the according indices $1 \leqslant i \leqslant s$, it holds by Theorem 3.6(1) resp. Theorem 3.7: $\lim \sup _{N \in J_{i}} f^{*}\left(v^{N}\right)=$ $\lim _{N \in J_{i}} f^{*}\left(v^{N}\right)=f^{*}\left(v_{0}\right)$ or $\lim \sup _{N \in J_{i}} f^{*}\left(v^{N}\right) \leqslant f^{*}\left(v_{0}\right)$, depending on whether $v_{0}$ belongs to ri $\left(\Phi_{i}\right)$ or $\operatorname{rb}\left(\Phi_{i}\right)$. Similarly it follows that $\lim \sup _{N \in J_{s+1}} f^{*}\left(v^{N}\right) \leqslant f^{*}\left(v_{0}\right)$. If the sequence $\left\{v^{N}\right\}$ contains infinitely many extremal points (thus the index set $J_{0}$ is infinite) then, by Theorem 3.4(3), it holds: $\lim \sup _{N \in J_{0}} f^{*}\left(v^{N}\right)=$ $\limsup _{N \in J_{0}} f\left(v^{N}\right)=\lim _{N \in J_{0}} f\left(v^{N}\right)=f\left(v_{0}\right)$. Combining these relations, we arrive at

$$
\limsup _{N \rightarrow \infty} f^{*}\left(v^{N}\right)=\operatorname{Max}\left\{\limsup _{N \in J_{i}} f^{*}\left(v^{N}\right) \mid 0 \leqslant i \leqslant s+1, J_{i} \text { infinite }\right\} \leqslant f^{*}\left(v_{0}\right) .
$$

Proof of Theorem 3.9. If $\mathrm{K}$ possesses at most countably many faces $\Phi_{s}$ of dimension $k \geqslant 1$ then, by Theorem 3.4(3) and Lemma 5.2, we get for all $v \in \mathrm{K}$ the decomposition

$$
f^{*}(v)=\mathbb{1}_{\mathrm{ext}(\mathrm{K})}(v) \cdot f(v)+\sum_{s=1}^{\infty} \mathbb{1}_{\mathrm{ri}\left(\Phi_{s}\right)}(v) \cdot f^{*}(v) .
$$

Here $\mathbb{1}_{\text {ext }(\mathrm{K})} \cdot f: \mathrm{K} \rightarrow \mathbb{R}$ belongs to the second Baire class (Lem. 5.4). For all $s, \mathbb{1}_{\mathrm{ri}\left(\Phi_{s}\right)}: \mathrm{K} \rightarrow \mathbb{R}$ admits a representation $\mathbb{1}_{\Phi_{s}}-\mathbb{1}_{\mathrm{rb}\left(\Phi_{s}\right)}$ as a difference of characteristic functions of compact sets, thus it remains within the first Baire class. The function $h_{s}: \mathrm{K} \rightarrow \mathbb{R}$ defined by

$$
h_{s}(v)=\left\{\begin{array}{l}
f^{*}(v) \mid v \in \operatorname{ri}\left(\Phi_{s}\right) \\
f(v) \mid v \in \mathrm{K} \backslash \operatorname{ri}\left(\Phi_{s}\right)
\end{array}\right.
$$


is upper semicontinuous since Theorem 3.6(1), Theorem 3.7 and $f^{*}(v) \leqslant f(v) \forall v \in \operatorname{rb}\left(\Phi_{s}\right)$ (Thm. 3.3(1)). Consequently, for all indices $s, \mathbb{1}_{\mathrm{ri}\left(\Phi_{s}\right)} \cdot h_{s} \equiv \mathbb{1}_{\mathrm{ri}\left(\Phi_{s}\right)} \cdot f^{*}: \mathrm{K} \rightarrow \mathbb{R}$ belongs to the first Baire class as well, and $f^{*} \mid \mathrm{K}$ must be a member of the second Baire class $\mathcal{B}^{2}(K, \mathbb{R})$.

\subsection{Lower semicontinuous extension $f^{\#}$ of $f^{*} \mid$ int $(\mathrm{K})$ to $\partial \mathrm{K}$}

In this section, we continue with the investigation of the envelope $f^{*}$. We will show that the limit $\lim _{v \rightarrow v_{0}, v \in \mathrm{R} \cap \operatorname{int}(\mathrm{K})} f^{*}(v)$ in the point $v_{0} \in \mathrm{R} \cap \partial \mathrm{K}$ exists along every ray $\mathrm{R}$ starting from the origin. This fact permits us to define a function $f^{\#}: \mathbb{R}^{n m} \rightarrow \overline{\mathbb{R}}$ in the following way:

Definition 3.11 ( $f^{\#}$ with modification of the boundary values of $\left.f^{*}\right)$. For $f \in \mathcal{F}_{\mathrm{K}}$, we define

$$
f^{\#}\left(v_{0}\right)=\left\{\begin{aligned}
f^{*}\left(v_{0}\right) \mid v_{0} & \in \operatorname{int}(\mathrm{K}) ; \\
\lim _{v \rightarrow v_{0}, v \in \mathrm{R} \cap \operatorname{int}(\mathrm{K})} f^{*}(v) \mid v_{0} & \in \partial \mathrm{K} ; \\
+\infty \mid v_{0} & \in \mathbb{R}^{n m} \backslash \mathrm{K} .
\end{aligned}\right.
$$

This definition establishes an analogy to the convex envelope $f^{c}$ which satisfies in all points $v_{0} \in \partial \mathrm{K}$ the radial limit relation $\lim _{v \rightarrow v_{0}, v \in \mathrm{R} \cap \operatorname{int}(\mathrm{K})} f^{c}(v)=f^{c}\left(v_{0}\right)^{48}$. Moreover, the function $f^{\#} \leqslant f^{*}$ is lower semicontinuous (Thm. 3.16). Consequently, $f^{\#}$ is the lower semicontinuous envelope of $f^{*}$, and its values on $\partial \mathrm{K}$ admit the radial limit representation according to Definition 3.11.

Theorem 3.12. Let $f \in \mathcal{F}_{\mathrm{K}}$ be given.

(1) $\left(\varepsilon-\delta\right.$ relation for $f^{*}$ along rays starting from the origin) Assume that the uniform continuity of $f$ on $\mathrm{K}$ is described through the $\varepsilon-\delta$ relation

$$
\left|v^{\prime}-v^{\prime \prime}\right| \leqslant \delta(\varepsilon)<1 \Longrightarrow\left|f\left(v^{\prime}\right)-f\left(v^{\prime \prime}\right)\right| \leqslant \varepsilon \quad \forall v^{\prime}, v^{\prime \prime} \in \mathrm{K} .
$$

Assume that two points $v, w \in \operatorname{int}(\mathrm{K})$ admit the following properties: (a) $v$, $w$ are situated on the same ray $\mathrm{R}$ starting from $\mathfrak{o}$, and $(b) 0<\operatorname{Dist}(w, \partial \mathrm{K})<\operatorname{Dist}(v, \partial \mathrm{K})<\frac{1}{2} c_{\mathrm{K}}$. Then $f^{*}$ obeys the following $\varepsilon-\delta$ estimate, which holds uniformly for all rays $\mathrm{R}$ starting from $\mathfrak{o}$ :

$$
\operatorname{Dist}(w, v) \leqslant \delta_{2}(\varepsilon) \Longrightarrow f^{*}(w)-f^{*}(v) \geqslant-2 \varepsilon
$$

with $\delta_{2}(\varepsilon)=\frac{1}{6} \delta(\varepsilon) \cdot c_{\mathrm{K}} / C_{\mathrm{K}}$ where $c_{\mathrm{K}}$ and $C_{\mathrm{K}}$ are the quantities defined in the beginning of the section.

(2) (Justification of Def. 3.11) Along every ray $\mathrm{R}$ starting from the origin, the following limit in the point $v_{0} \in$ $\mathrm{R} \cap \partial \mathrm{K}$ exists:

$$
\lim _{v \rightarrow v_{0}, v \in \mathrm{R} \cap \operatorname{int}(\mathrm{K})} f^{*}(v) .
$$

(3) $\left(\varepsilon-\delta\right.$ relation for $f^{\#}$ along rays starting from the origin) Under the assumptions of Part (1), we consider two points $v, w \in \mathrm{K}$ which (a) are situated on the same ray $\mathrm{R}$ starting from $\mathfrak{o}$ and (b) satisfy $0 \leqslant$ $\operatorname{Dist}(w, \partial \mathrm{K}) \leqslant \operatorname{Dist}(v, \partial \mathrm{K})<\frac{1}{2} c_{\mathrm{K}}$. Then the $\varepsilon-\delta$ estimate from Part (1) can be extended to $f^{\#}$ :

$$
\operatorname{Dist}(w, v) \leqslant \delta_{2}(\varepsilon) \Longrightarrow f^{\#}(w)-f^{\#}(v) \geqslant-2 \varepsilon,
$$

and again the estimate holds uniformly for all rays $\mathrm{R}$ starting from $\mathfrak{o}$.

Lemma 3.13 (Inequalities between $f^{(q c)}, f^{\#}$ and $f^{*}$ ). For all $f \in \mathcal{F}_{\mathrm{K}}$ it holds:

$$
f^{(q c)}(v) \leqslant f^{\#}(v) \leqslant f^{*}(v) \quad \forall v \in \mathbb{R}^{n m} .
$$

\footnotetext{
$48[34]$, p. 59 , Theorem 2.35 .
} 


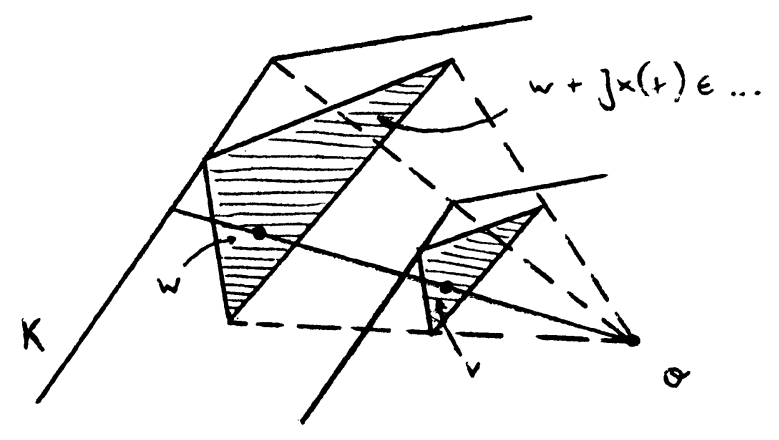

FigURE 4.

Theorem 3.14 (Continuity of $\left.f^{\#}\right)^{49}$. Let $f \in \mathcal{F}_{\mathrm{K}}$ be given.

(1) $f^{\#}$ is continuous in every point $v \in \operatorname{int}(\mathrm{K})$.

(2) $f^{\#} \mid \mathrm{K}$ is continuous in every point $v \in \operatorname{ext}(\mathrm{K})$, and there it holds $f^{\#}(v)=f^{*}(v)=f(v)$.

(3) If $\partial \mathrm{K}=\operatorname{ext}(\mathrm{K})$ then $f^{\#} \mid \mathrm{K}$ is continuous on the whole set $\mathrm{K}$, and $f^{\#}$ belongs to $\mathcal{F}_{\mathrm{K}}$.

The proof of the lower semicontinuity of $f^{\#}$ is based on the following proposition:

Theorem $3.15\left(\varepsilon-\delta\right.$ relation for $f^{\#}$ in points $\left.v \in \partial \mathrm{K}\right)$. Let a function $f \in \mathcal{F}_{\mathrm{K}}$ and a point $v \in \partial \mathrm{K}$ be given Then for arbitrary $\varepsilon>0$ there exists $\delta_{4}(\varepsilon, v)$ with

$$
\operatorname{Dist}(w, v) \leqslant \delta_{4}(\varepsilon, v) \Longrightarrow f^{\#}(w)-f^{\#}(v) \geqslant-5 \varepsilon \quad \forall w \in \mathrm{K} .
$$

Theorem 3.16 (Lower semicontinuity of $f^{\#}$ ). Again, let a function $f \in \mathcal{F}_{\mathrm{K}}$ be given.

(1) The function $f^{\#}$ is lower semicontinuous.

(2) For all $v_{0} \in \mathbb{R}^{n m}$, it holds that $f^{\#}\left(v_{0}\right)=\liminf _{v \rightarrow v_{0}} f^{*}(v)$. Consequently, $f^{\#}$ is the lower semicontinuous envelope of $f^{*}$.

Proof of Theorem 3.12. (1) Let Dist $(\mathfrak{o}, v)=D$ and Dist $(\mathfrak{o}, w)=D+d$. Then it holds that:

$$
0<\frac{c_{\mathrm{K}}}{2} \leqslant D<D+d<C_{\mathrm{K}} \Longrightarrow \frac{c_{\mathrm{K}}}{2 C_{\mathrm{K}}}<\frac{c_{\mathrm{K}}}{2(D+d)} \leqslant \frac{D}{D+d}<1,
$$

and the points $v$ and $w$ can be represented as

$$
v=\frac{D}{D+d} w \quad \text { resp. } \quad w=\frac{D+d}{D} v .
$$

Choose a number $\varepsilon>0$. There exists a function $x \in W_{0}^{1, \infty}\left(\Omega, \mathbb{R}^{n}\right)$ with

$$
f^{*}(w)+\varepsilon=f^{*}\left(\frac{D+d}{D} v\right)+\varepsilon \geqslant \frac{1}{|\Omega|} \int_{\Omega} f\left(\frac{D+d}{D} v+J x(t)\right) \mathrm{d} t
$$

and

$$
\frac{D+d}{D} v+J x(t) \in \mathrm{K}(\forall) t \in \Omega \quad \text { resp. } \quad v+\frac{D}{D+d} J x(t) \in \frac{D}{D+d} \mathrm{~K} \subset \mathrm{K}(\forall) t \in \Omega
$$

(cf. Fig. 4).

\footnotetext{
${ }^{49} C f$. Theorem 3.6.
} 
As in the proof of Theorem 3.5, it holds further that $|J x(t)| \leqslant 2 C_{\mathrm{K}}(\forall) t \in \Omega$. We get

$\left|\left(\frac{D+d}{D} v+J x(t)\right)-\left(v+\frac{D}{D+d} J x(t)\right)\right| \leqslant d\left(\frac{|v|}{D}+\frac{|J x(t)|}{D+d}\right) \leqslant \frac{d}{D}(|v|+|J x(t)|) \leqslant d \cdot \frac{6 C_{\mathrm{K}}}{c_{\mathrm{K}}}$.

Thus, defining $\delta_{2}(\varepsilon)=\frac{1}{6} \delta(\varepsilon) \cdot c_{\mathrm{K}} / C_{\mathrm{K}}$, we find

$$
|w-v|=d \leqslant \delta_{2}(\varepsilon) \Longrightarrow\left|f\left(\frac{D+d}{D} v+J x(t)\right)-f\left(v+\frac{D}{D+d} J x(t)\right)\right| \leqslant \varepsilon
$$

and

$$
\begin{aligned}
f^{*}(w)+\varepsilon & \geqslant \frac{1}{|\Omega|} \int_{\Omega} f\left(\frac{D+d}{D} v+J x(t)\right) \mathrm{d} t \\
& \geqslant-\frac{1}{|\Omega|} \int_{\Omega}\left|f\left(\frac{D+d}{D} v+J x(t)\right)-f\left(v+\frac{D}{D+d} J x(t)\right)\right| \mathrm{d} t+\frac{1}{|\Omega|} \int_{\Omega} f\left(v+\frac{D}{D+d} J x(t)\right) \mathrm{d} t \\
& \geqslant-\varepsilon+f^{*}(v) .
\end{aligned}
$$

(2) Since the function $f^{*}$ is bounded on int $(\mathrm{K})$, the numbers $L^{\prime}=\limsup _{v \rightarrow v_{0}, v \in \mathrm{R} \cap \operatorname{int}(\mathrm{K})} f^{*}(v)$ and $L^{\prime \prime}=$ $\liminf _{v \rightarrow v_{0}, v \in \mathrm{R} \cap \operatorname{int}(\mathrm{K})} f^{*}(v)$ must be finite. We choose sequences $\left\{v^{N}\right\}, \mathrm{R} \cap \operatorname{int}(\mathrm{K}) \rightarrow v_{0}$ and $\left\{w^{N}\right\}$, $\mathrm{R} \cap \operatorname{int}(\mathrm{K}) \rightarrow v_{0}$ with

$$
\begin{aligned}
& \left|v^{N}-v_{0}\right| \leqslant \frac{1}{N} ; \quad\left|f^{*}\left(v^{N}\right)-L^{\prime}\right| \leqslant \frac{1}{N} ; \\
& \left|w^{N}-v_{0}\right| \leqslant \frac{1}{N} ; \quad\left|f^{*}\left(w^{N}\right)-L^{\prime \prime}\right| \leqslant \frac{1}{N} \quad \text { as well as } \\
& \left|w^{N}-v^{N}\right| \leqslant \frac{2}{N}
\end{aligned}
$$

for all $N \in \mathbb{N}$ (the last property can be assured, if necessary, by passage to subsequences). Since $L^{\prime} \geqslant L^{\prime \prime}$, it follows:

$$
|| f^{*}\left(v^{N}\right)-f^{*}\left(w^{N}\right)|-| L^{\prime}-L^{\prime \prime}|| \leqslant\left|\left(f^{*}\left(v^{N}\right)-L^{\prime}\right)-\left(f^{*}\left(w^{N}\right)-L^{\prime \prime}\right)\right| \leqslant \frac{2}{N}
$$

and

$$
\left(L^{\prime}-L^{\prime \prime}\right)-\frac{2}{N} \leqslant\left|f^{*}\left(v^{N}\right)-f^{*}\left(w^{N}\right)\right| \leqslant\left(L^{\prime}-L^{\prime \prime}\right)+\frac{2}{N} .
$$

Assume that $L^{\prime}-L^{\prime \prime}>0$. Then for all $N$ with $2 / N<\left(L^{\prime}-L^{\prime \prime}\right)$, it holds even that

$$
\begin{aligned}
& \left(L^{\prime}-L^{\prime \prime}\right)-\frac{2}{N} \leqslant f^{*}\left(v^{N}\right)-f^{*}\left(w^{N}\right) \leqslant\left(L^{\prime}-L^{\prime \prime}\right)+\frac{2}{N} \text { resp. } \\
& \frac{2}{N}-\left(L^{\prime}-L^{\prime \prime}\right) \geqslant f^{*}\left(w^{N}\right)-f^{*}\left(v^{N}\right) \geqslant-\frac{2}{N}-\left(L^{\prime}-L^{\prime \prime}\right) .
\end{aligned}
$$

Let $0<\varepsilon<\frac{3}{8}\left(L^{\prime}-L^{\prime \prime}\right)$ be given. By Part (1), there exists a $0<\delta_{2}(\varepsilon)<1$ and thus a $N(\varepsilon) \in \mathbb{N}$ with $2 / N(\varepsilon)<\left(L^{\prime}-L^{\prime \prime}\right)$ such that

$$
\left|w^{N}-v^{N}\right| \leqslant \frac{2}{N} \leqslant \delta_{2}(\varepsilon) \Longrightarrow f^{*}\left(w^{N}\right)-f^{*}\left(v^{N}\right) \geqslant-2 \varepsilon>-\frac{3}{4}\left(L^{\prime}-L^{\prime \prime}\right) \quad \forall N \geqslant N(\varepsilon) .
$$

Summing up, we arrive at a contradiction since

$$
0 \geqslant f^{*}\left(w^{N}\right)-f^{*}\left(v^{N}\right)>-\frac{3}{4}\left(L^{\prime}-L^{\prime \prime}\right)>\frac{2}{N}-\left(L^{\prime}-L^{\prime \prime}\right) \geqslant f^{*}\left(w^{N}\right)-f^{*}\left(v^{N}\right)
$$

for all $N \geqslant N(\varepsilon)$ sufficiently large. Thus the equality $L^{\prime}=L^{\prime \prime}$ holds. 
(3) We have to examine only the case where $w \neq v$ belongs to $\mathrm{R} \cap \partial \mathrm{K}$. Then we find a sequence of points $\left\{w^{N}\right\}, \mathrm{R} \cap \operatorname{int}(\mathrm{K}) \rightarrow w$ with $\operatorname{Dist}\left(w^{N}, \partial \mathrm{K}\right) \leqslant \operatorname{Dist}(v, \partial \mathrm{K})$ for all $N$. For all points within this sequence, it holds that $\operatorname{Dist}\left(w^{N}, v\right) \leqslant \operatorname{Dist}(w, v) \leqslant \delta_{2}(\varepsilon)$, and by Part $(1)$ we get:

$$
f^{\#}\left(w^{N}\right)-f^{\#}(v)=f^{*}\left(w^{N}\right)-f^{*}(v) \geqslant-2 \varepsilon \Longrightarrow f^{\#}(w)-f^{\#}(v)=\lim _{N \rightarrow \infty} f^{\#}\left(w^{N}\right)-f^{\#}(v) \geqslant-2 \varepsilon
$$

Proof of Lemma 3.13. In view to Theorem 3.3(1), the inequalities have to be proven only for $v_{0} \in \partial \mathrm{K}$. Denote the ray $\overrightarrow{\mathfrak{o} v_{0}}$ by $\mathrm{R}$. By Theorem 2.18(2), Theorem 3.3(1) and Theorem 3.7, for any sequence $\left\{v^{N}\right\}, \mathrm{R} \rightarrow v_{0}$ it holds that:

$$
\begin{aligned}
f^{(q c)}\left(v_{0}\right) \leqslant \liminf _{v \rightarrow v_{0}} f^{(q c)}(v) \leqslant \liminf _{N \rightarrow \infty} f^{(q c)}\left(v^{N}\right) \leqslant \liminf _{N \rightarrow \infty} f^{*}\left(v^{N}\right) & =\lim _{N \rightarrow \infty} f^{*}\left(v^{N}\right) \\
& =f^{\#}\left(v_{0}\right)=\limsup _{N \rightarrow \infty} f^{*}\left(v^{N}\right) \leqslant f^{*}\left(v_{0}\right) .
\end{aligned}
$$

Proof of Theorem 3.14. The assertion is an immediate consequence of Theorem 2.11.

Proof of Theorem 3.15. Choose a number $\varepsilon>0$. By Theorem 3.12(2), there exists a point $v^{\prime} \in \mathrm{R}_{v}$ on the ray $\mathrm{R}_{v}=\overrightarrow{\mathfrak{o} v}$ with

$$
0<\operatorname{Dist}\left(v^{\prime}, \partial \mathrm{K}\right) \leqslant \operatorname{Min}\left(1, \frac{\delta_{2}(\varepsilon)}{2}, \frac{c_{\mathrm{K}}}{2}\right) \quad \text { and }\left|f^{\#}(v)-f^{\#}\left(v^{\prime}\right)\right| \leqslant \varepsilon
$$

Define $\delta_{3}(\varepsilon, v)=\operatorname{Dist}\left(v^{\prime}, \partial \mathrm{K}\right)$. Since the distance function Dist $(\cdot, \partial \mathrm{K})$ is Lipschitz continuous with constant 1 $([9]$, p. 50$)$, for all $w^{\prime} \in \mathrm{K}\left(v^{\prime}, \frac{1}{2} \delta_{1}(\varepsilon) \delta_{3}(\varepsilon, v)\right)$ it follows:

$$
\begin{aligned}
\mid \operatorname{Dist} & \left(w^{\prime}, \partial \mathrm{K}\right)-\operatorname{Dist}\left(v^{\prime}, \partial \mathrm{K}\right)|\leqslant| w^{\prime}-v^{\prime} \mid \leqslant \frac{1}{2} \delta_{1}(\varepsilon) \delta_{3}(\varepsilon, v) \Longrightarrow \\
& -\frac{1}{2} \delta_{1}(\varepsilon) \delta_{3}(\varepsilon, v) \leqslant \operatorname{Dist}\left(w^{\prime}, \partial \mathrm{K}\right)-\operatorname{Dist}\left(v^{\prime}, \partial \mathrm{K}\right) \Longrightarrow \\
& -\frac{1}{2} \delta_{1}(\varepsilon) \delta_{3}(\varepsilon, v)+\operatorname{Dist}\left(v^{\prime}, \partial \mathrm{K}\right)=\delta_{3}(\varepsilon, v)\left(1-\frac{\delta_{1}(\varepsilon)}{2}\right) \leqslant \operatorname{Dist}\left(w^{\prime}, \partial \mathrm{K}\right) .
\end{aligned}
$$

Since $\delta_{1}(\varepsilon) \leqslant 1$, we conclude:

$\operatorname{Min}\left(1, \operatorname{Dist}\left(v^{\prime}, \partial \mathrm{K}\right), \operatorname{Dist}\left(w^{\prime}, \partial \mathrm{K}\right)\right)$

$$
\geqslant \operatorname{Min}\left(1, \delta_{3}(\varepsilon, v), \delta_{3}(\varepsilon, v)\left(1-\frac{\delta_{1}(\varepsilon)}{2}\right)\right)=\delta_{3}(\varepsilon, v)\left(1-\frac{\delta_{1}(\varepsilon)}{2}\right) \geqslant \frac{\delta_{3}(\varepsilon, v)}{2} .
$$

From Theorem 3.5 we get

$$
\left|v^{\prime}-w^{\prime}\right| \leqslant \delta_{1}(\varepsilon) \cdot \frac{\delta_{3}(\varepsilon, v)}{2} \leqslant \delta_{1}(\varepsilon) \cdot \operatorname{Min}\left(1, \operatorname{Dist}\left(v^{\prime}, \partial \mathrm{K}\right), \operatorname{Dist}\left(w^{\prime}, \partial \mathrm{K}\right)\right) \Longrightarrow\left|f^{\#}\left(v^{\prime}\right)-f^{\#}\left(w^{\prime}\right)\right| \leqslant 2 \varepsilon
$$

Consider now the points $w \in \mathrm{K}$ with $|v-w| \leqslant \frac{1}{2} \delta_{1}(\varepsilon) \delta_{3}(\varepsilon, v)=\delta_{4}(\varepsilon, v)$. By the intercept theorems, for any of these points $w$ there exists a further point $w^{\prime} \in \mathrm{R}_{w} \cap \operatorname{int}(\mathrm{K})$ on the ray $\mathrm{R}_{w}=\overrightarrow{\mathfrak{o} w}$ such that $w^{\prime}$ belongs at the same time to $\mathrm{K}\left(v^{\prime}, \frac{1}{2} \delta_{1}(\varepsilon) \delta_{3}(\varepsilon, v)\right)$ (cf. Fig. 5). 


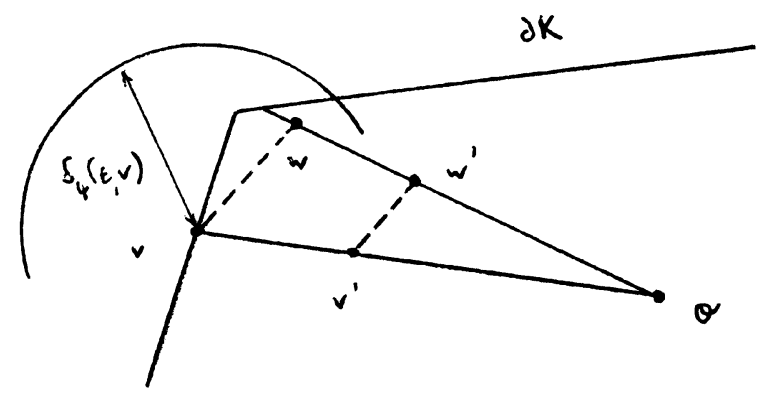

Figure 5.

For such a point $w^{\prime}$, it holds:

$$
\begin{aligned}
\left|w-w^{\prime}\right| \leqslant|w-v|+\left|v-v^{\prime}\right|+\left|v^{\prime}-w^{\prime}\right| & \leqslant \frac{1}{2} \delta_{1}(\varepsilon) \delta_{3}(\varepsilon, v)+\delta_{3}(\varepsilon, v)+\frac{1}{2} \delta_{1}(\varepsilon) \delta_{3}(\varepsilon, v) \\
& =\delta_{3}(\varepsilon, v)\left(1+\delta_{1}(\varepsilon)\right) \leqslant 2 \delta_{3}(\varepsilon, v) \leqslant \delta_{2}(\varepsilon)
\end{aligned}
$$

thus we get from Theorem 3.12(1) and (3): $f^{\#}(w)-f^{\#}\left(w^{\prime}\right) \geqslant-2 \varepsilon$. Summing up, we get for all $w \in \mathrm{K}$ the implication: From $|v-w| \leqslant \delta_{4}(\varepsilon, v)$ it follows that

$$
\begin{aligned}
& -2 \varepsilon \leqslant f^{\#}(w)-f^{\#}\left(w^{\prime}\right) ;-2 \varepsilon \leqslant f^{\#}\left(w^{\prime}\right)-f^{\#}\left(v^{\prime}\right) ;-\varepsilon \leqslant f^{\#}(v)-f^{\#}\left(v^{\prime}\right) \Longrightarrow \\
& -5 \varepsilon \leqslant f^{\#}(w)-f^{\#}(v) .
\end{aligned}
$$

Proof of Theorem 3.16. (1) In view of Theorem 3.14(1), the lower semicontinuity remains to prove for points $v_{0} \in \partial \mathrm{K}$ only. Thus we consider a sequence $\left\{v^{N}\right\}, \mathbb{R}^{n m} \rightarrow v_{0} \in \partial \mathrm{K}$. For an infinite subsequence $\left\{v^{N}\right\}_{N \in J_{0}}$ with members $v^{N} \in \mathbb{R}^{n m} \backslash \mathrm{K}$, it holds that $\liminf \inf _{N \in J_{0}} f^{\#}\left(v^{N}\right)=+\infty>f^{\#}\left(v_{0}\right)$. For an infinite subsequence $\left\{v^{N}\right\}_{N \in J_{1}}$ with members $v^{N} \in \mathrm{K}$, we have by Theorem 3.15:

$$
\begin{aligned}
\forall \varepsilon>0 \exists N(\varepsilon) \in J_{1} \forall N \geqslant N(\varepsilon), N \in J_{1}:\left|v^{N}-v_{0}\right| \leqslant \delta_{4}\left(\varepsilon, v_{0}\right) \Longrightarrow \\
f^{\#}\left(v^{N}\right) \geqslant f^{\#}\left(v_{0}\right)-5 \varepsilon \Longrightarrow \liminf _{N \in J_{1}} f^{\#}\left(v^{N}\right) \geqslant f^{\#}\left(v_{0}\right)-5 \varepsilon .
\end{aligned}
$$

Since $\varepsilon>0$ was arbitrary, we may conclude that $\liminf _{N \in J_{1}} f^{\#}\left(v^{N}\right) \geqslant f^{\#}\left(v_{0}\right)$.

(2) Again, the assertion must be proven for $v_{0} \in \partial \mathrm{K}$ only. Denote the ray $\overrightarrow{\mathfrak{o} v_{0}}$ by $\mathrm{R}$. Then from Part $(1)$ and Lemma 3.13, it follows:

$$
f^{\#}\left(v_{0}\right) \leqslant \liminf _{v \rightarrow v_{0}} f^{\#}(v) \leqslant \liminf _{v \rightarrow v_{0}} f^{*}(v) \leqslant \lim _{v \rightarrow v_{0}, v \in \mathrm{R} \cap \operatorname{int}(\mathrm{K})} f^{*}(v)=f^{\#}\left(v_{0}\right) .
$$

\subsection{Morrey's integral inequality for $f^{*}$ and $f^{\#}$}

In spite of the formal analogy between the formulae (2.10) from Theorem 2.8. and (3.1) in Definition 3.1, we cannot prove, in general, that $f^{*}$ is quasiconvex. On the one hand, even the proof of Borel measurability of $f^{*} \mid \mathrm{K}$ is missed as soon as $\mathrm{K}$ possesses uncountably many faces of dimension $k \geqslant 1$ ( $c f$. Thm. 3.9). On the other hand, even when the Borel measurability of $f^{*} \mid \mathrm{K}$ is explicitly assumed, the validity of Morrey's integral inequality can be confirmed for points $v \in \operatorname{int}(\mathrm{K})$ only. The reason is that in the process of approximation of 
$x \in W_{0}^{1, \infty}\left(\Omega, \mathbb{R}^{n}\right)$ by piecewise affine functions $w^{N}$, the control restriction $v+J w^{N}(t) \in \mathrm{K}(\forall) t \in \Omega$ can be conserved only under the additional assumption that $v+J x(t) \in(1-\gamma) \mathrm{K}, 0<\gamma<1^{50}$. We summarize the according results of Dacorogna/Marcellini in Theorem 3.17:

Theorem 3.17 (Morrey's integral inequality for $\left.f^{*}\right)^{51}$. Let a function $f \in \mathcal{F}_{\mathrm{K}}$ be given .

(1) For all $v \in \operatorname{int}(\mathrm{K})$ and all $0<\gamma<1$, it holds that:

$$
f^{*}(v)=\inf \left\{\frac{1}{|\Omega|} \int_{\Omega} f^{*}(v+J x(t)) \mathrm{d} t \mid x \in W_{0}^{1, \infty}\left(\Omega, \mathbb{R}^{n}\right), v+J x(t) \in(1-\gamma) \mathrm{K}(\forall) t \in \Omega\right\} .
$$

(2) If $f^{*} \mid \mathrm{K}$ is Borel measurable then Morrey's integral inequality is valid for all $v \in$ int $(\mathrm{K})$ :

$$
f^{*}(v)=\inf \left\{\frac{1}{|\Omega|} \int_{\Omega} f^{*}(v+J x(t)) \mathrm{d} t \mid x \in W_{0}^{1, \infty}\left(\Omega, \mathbb{R}^{n}\right), v+J x(t) \in \mathrm{K}(\forall) t \in \Omega\right\} .
$$

Remark. Actually, in [14], p. 31 ff., only Part (1) has been proven. Assuming, however, the Borel measurability of $f^{*} \mid \mathrm{K}$ explicitly, Part (2) follows from Part (1) together with Theorem 3.7 since the existence of the integral $\int_{\Omega} f^{*}(v+J x(t)) \mathrm{d} t$ is then assured if $v+J x(t)$ take values in $\partial \mathrm{K}$ as well.

From Theorem 3.17(2), the quasiconvexity of $f^{*}$ follows only in the special case $\partial \mathrm{K}=$ ext $(\mathrm{K})$. The function $f^{\#}$ however, turns out to be, independently of the facial structure of $\partial \mathrm{K}$, always a quasiconvex and rank one convex function. In Theorem $4.5(3)$ below we provide an example showing that $f^{*}$, in general, may even be not rank one convex.

Theorem 3.18 (Quasiconvexity and rank one convexity of $f^{*}$ for $\partial \mathrm{K}=\operatorname{ext}(\mathrm{K})$ ). Let a function $f \in \mathcal{F}_{\mathrm{K}}$ be given. If $\partial \mathrm{K}=\operatorname{ext}(\mathrm{K})$ then the function $f^{*}: \mathbb{R}^{n m} \rightarrow \overline{\mathbb{R}}$ is quasiconvex as well as rank one convex.

Theorem 3.19 (Quasiconvexity and rank one convexity of $f^{\#}$ ). Let a function $f \in \mathcal{F}_{\mathrm{K}}$ be given. Then the function $f^{\#}: \mathbb{R}^{n m} \rightarrow \overline{\mathbb{R}}$ is quasiconvex as well as rank one convex.

Proof of Theorem 3.17. (2) Let a point $v \in \operatorname{int}(\mathrm{K})$ and a function $x \in W_{0}^{1, \infty}(\Omega)$ with $v+J x(t) \in \mathrm{K}(\forall) t \in \Omega$ be given. Then it holds for all $N \in \mathbb{N}$ :

$$
f^{*}(v) \leqslant \frac{1}{|\Omega|} \int_{\Omega} f^{*}\left(v+\frac{N}{N+1} J x(t)\right) \mathrm{d} t,
$$

and from Fatou's lemma ([21], p. 143, Lem. 5.1, and p. 149, 5.4) and Theorem 3.7 it follows:

$$
\begin{aligned}
& f^{*}(v) \leqslant \limsup _{N \rightarrow \infty} \frac{1}{|\Omega|} \int_{\Omega} f^{*}\left(v+\frac{N}{N+1} J x(t)\right) \mathrm{d} t \leqslant \frac{1}{|\Omega|} \int_{\Omega} \limsup _{N \rightarrow \infty} f^{*}\left(v+\frac{N}{N+1} J x(t)\right) \mathrm{d} t \\
& \leqslant \frac{1}{|\Omega|} \int_{\Omega} f^{*}(v+J x(t)) \mathrm{d} t .
\end{aligned}
$$

By assumption on $f^{*} \mid \mathrm{K}$, the existence of the last integral is assured. Then we find

$$
f^{*}(v)=\inf \left\{\frac{1}{|\Omega|} \int_{\Omega} f^{*}(v+J x(t)) \mathrm{d} t \mid x \in W_{0}^{1, \infty}\left(\Omega, \mathbb{R}^{n}\right), v+J x(t) \in \mathrm{K}(\forall) t \in \Omega\right\},
$$

and together with Theorem 2.11(2), we arrive at Morrey's integral inequality.

Proof of Theorem 3.18. If $\partial \mathrm{K}=\operatorname{ext}(\mathrm{K})$ then, by Theorem 3.6(3), $f^{*}$ belongs to $\mathcal{F}_{\mathrm{K}}$, and the conditions (1) and (2) from Definition 2.9 are satisfied. By Theorem 3.17(2), Morrey's integral inequality holds in all points

\footnotetext{
${ }^{50}[42]$, p. 34 f., Proof of Theorem 3.17, Step 2.

51 Specification of [14], p. 27, Theorem 7.2.
} 
$v \in \operatorname{int}(\mathrm{K})$, and by Theorem 2.11(2), it is automatically satisfied in the boundary points $v \in \partial \mathrm{K}=\operatorname{ext}(\mathrm{K})$. By Theorem 2.11(2), $f^{*}$ is quasiconvex, and by Theorem 2.12, the restriction $f^{*} \mid \operatorname{int}(\mathrm{K})$ is rank one convex. Since $f^{*} \in \mathcal{F}_{\mathrm{K}}$ and $\partial \mathrm{K}=\operatorname{ext}(\mathrm{K})$, Jensen's inequality can be extended to arbitrary $v, w \in \mathrm{K}$ with $\operatorname{Rg}(v-w) \leqslant 1$ (considered as $(n, m)$-matrices).

Proof of Theorem 3.19. We observe first that $f^{\#} \mid \mathrm{K}$ is bounded and, as a restriction of a lower semicontinuous function (Thm. 3.16(1)) to a closed set (see [7], p. 136, Thm. 1), it is lower semicontinuous as well and belongs to the first Baire class. Consequently, $f_{\#}^{\#}$ satisfies the conditions (1) and (2) from Definition 2.9. Let now $v_{0} \in \mathrm{K}$ be given. To a function $x \in W_{0}^{1, \infty}\left(\Omega, \mathbb{R}^{n}\right)$ with $v_{0}+J x(t) \in \mathrm{K}(\forall) t \in \Omega$, we define

$$
v^{N}=\frac{N-1}{N} v_{0} \text { and } x^{N}(t)=\frac{N-1}{N} x(t) .
$$

Then all points $v^{N}$ belong to int $(\mathrm{K})$, and we have $v^{N}+J x^{N}(t) \in \operatorname{int}(\mathrm{K})(\forall) t \in \Omega$. By Definition 3.11 and Theorem 3.17(2), it holds:

$$
f^{\#}\left(v^{N}\right)=f^{*}\left(v^{N}\right) \leqslant \frac{1}{|\Omega|} \int_{\Omega} f^{*}\left(v^{N}+J x^{N}(t)\right) \mathrm{d} t=\frac{1}{|\Omega|} \int_{\Omega} f^{\#}\left(v^{N}+J x^{N}(t)\right) \mathrm{d} t \quad \forall N \in \mathbb{N} .
$$

Obviously, it holds that $\lim _{N \rightarrow \infty}\left(v^{N}+J x^{N}(t)\right)=v_{0}+J x(t) \forall t \in \Omega$. If $t \in \Omega$ is such that $v_{0}+J x(t)$ belongs to $\mathrm{K}$ then this relation gives pointwise convergence along the according ray starting from $\mathfrak{o}$, and we get

$$
\lim _{N \rightarrow \infty} f^{\#}\left(v^{N}+J x^{N}(t)\right)=\lim _{N \rightarrow \infty} f^{*}\left(v^{N}+J x^{N}(t)\right)=f^{\#}\left(v_{0}+J x(t)\right) \quad(\forall) t \in \Omega .
$$

From inequality (3.90), we derive with Lebesgue's convergence theorem:

$$
\lim _{N \rightarrow \infty} f^{\#}\left(v^{N}\right)=f^{\#}\left(v_{0}\right) \leqslant \frac{1}{|\Omega|} \int_{\Omega} \lim _{N \rightarrow \infty} f^{\#}\left(v^{N}+J x^{N}(t)\right) \mathrm{d} t=\frac{1}{|\Omega|} \int_{\Omega} f^{\#}\left(v_{0}+J x(t)\right) \mathrm{d} t .
$$

Then from Theorem 2.11(2), the quasiconvexity of $f_{\#}^{\#}$ follows. In order to prove its rank one convexity as well, we choose a number $0 \leqslant \lambda \leqslant 1$ and points $v, w \in \mathrm{K}$ (considered as $(n, m)$-matrices) with $\operatorname{Rg}(v-w) \leqslant 1$. Define $v_{0}=\lambda v+(1-\lambda) w$. Then on the three rays $\mathrm{R}_{v}=\overrightarrow{\mathfrak{o} v}, \mathrm{R}_{w}=\overrightarrow{\mathfrak{o} w}$ and $\mathrm{R}_{0}=\overrightarrow{\mathfrak{o} v_{0}}$, the relations

$$
f^{\#}(v)=\lim _{\mu \rightarrow 1-0} f^{\#}(\mu v), \quad f^{\#}(w)=\lim _{\mu \rightarrow 1-0} f^{\#}(\mu w) \quad \text { and } \quad f^{\#}\left(v_{0}\right)=\lim _{\mu \rightarrow 1-0} f^{\#}\left(\mu v_{0}\right)
$$

hold. Since $\mu v, \mu w$ and $\mu v_{0}$ belong to int (K) for all $0<\mu<1$, we derive with Theorem 2.12 from boundedness and quasiconvexity of $f^{\#}$ :

$$
\begin{aligned}
f^{\#}\left(\mu v_{0}\right) & \leqslant \lambda f^{\#}(\mu v)+(1-\lambda) f^{\#}(\mu w) \Longrightarrow f^{\#}\left(v_{0}\right)=\lim _{\mu \rightarrow 1-0} f^{\#}\left(\mu v_{0}\right) \\
& \leqslant \lambda \cdot \lim _{\mu \rightarrow 1-0} f^{\#}(\mu v)+(1-\lambda) \cdot \lim _{\mu \rightarrow 1-0} f^{\#}(\mu w)=\lambda f^{\#}(v)+(1-\lambda) f^{\#}(w) .
\end{aligned}
$$

\section{The Representation theOREM For $f^{(q c)}$}

\subsection{The representation theorem and its corollaries}

In consequence of Theorem 3.16(1) and Theorem 3.19, the function $f^{\#}$ is lower semicontinuous and quasiconvex and thus feasible in the forming of $f^{(q c)}$ according to Definition 2.14(2). We will prove now that, for $f \in \mathcal{F}_{\mathrm{K}}$, the functions $f^{\#}$ and $f^{(q c)}$ are even identical. 
Theorem 4.1 (First representation theorem for $\left.f^{(q c)}\right)$. Let a function $f \in \mathcal{F}_{\mathrm{K}}$ be given. Then the function $f^{\#}$ from Definition 3.11 and the lower semicontinuous quasiconvex envelope $f^{(q c)}$ coincide. Consequently, $f^{(q c)}: \mathbb{R}^{n m} \rightarrow \overline{\mathbb{R}}$ admits the representation

$$
f^{(q c)}\left(v_{0}\right)=\left\{\begin{aligned}
f^{*}\left(v_{0}\right) \mid & v_{0} \in \operatorname{int}(\mathrm{K}) ; \\
\lim _{v \rightarrow v_{0}, v \in \mathrm{R} \cap \operatorname{int}(\mathrm{K})} f^{*}(v) \mid v_{0} & \in \partial \mathrm{K} ; \\
+\infty \mid v_{0} & \in \mathbb{R}^{n m} \backslash \mathrm{K} .
\end{aligned}\right.
$$

In view of the definition of $f^{*}$, Theorem 4.1. can be seen as a generalization of Dacorogna's representation theorem for the quasiconvex envelope (Thm. 2.8). As a corollary, we infer that not only $f^{(q c)} \mid \operatorname{int}(\mathrm{K})$ is rank one convex but also $f^{(q c)}$ itself.

Theorem 4.2. Let a function $f \in \mathcal{F}_{\mathrm{K}}$ be given .

(1) (Rank one convexity of $f^{(q c)}$ ) Then the function $f^{(q c)}: \mathbb{R}^{n m} \rightarrow \overline{\mathbb{R}}$ is rank one convex.

(2) (Inequalities between $f^{c}, f^{(q c)}, f^{r c}$ and $f$ ) For all $v \in \mathbb{R}^{n m}$, the following inequalities hold:

$$
f^{c}(v) \leqslant f^{(q c)}(v) \leqslant f^{r c}(v) \leqslant f(v) .
$$

We point out that, in the special cases $n=1$ or $m=1$, the generalized convex envelopes coincide.

Theorem 4.3 (Coincidence of $f^{c}$ and $f^{(q c)}$ for $n=1$ or $m=1$ ). Let a convex body $\mathrm{K} \subset \mathbb{R}^{n m}$ and a function $f \in \mathcal{F}_{\mathrm{K}}$ be given. If $n=1$ or $m=1$ then the envelopes $f^{c}, f^{(q c)}$ and $f^{r c}$ are identical.

Proof of Theorem 4.1. Let $g: \mathbb{R}^{n m} \rightarrow \overline{\mathbb{R}}$ be some lower semicontinuous quasiconvex function with $g(v) \leqslant f(v)$ $\forall v \in \mathbb{R}^{n m}$. By Theorem 3.3(2) we have for all points $v \in \mathbb{R}^{n m} \backslash \partial \mathrm{K}$ :

$$
g(v) \leqslant f^{*}(v)=f^{\#}(v) .
$$

Consider now a point $v_{0} \in \partial \mathrm{K}$ and the ray $\mathrm{R}=\overrightarrow{\mathfrak{o} v_{0}}$. Since $g$ is lower semicontinuous, we find along every sequence $\left\{v^{N}\right\}, \mathrm{R} \rightarrow v_{0}$ :

$$
g\left(v_{0}\right) \leqslant \liminf _{v \rightarrow v_{0}} g(v) \leqslant \liminf _{N \rightarrow \infty} g\left(v^{N}\right) \leqslant \liminf _{N \rightarrow \infty} f^{*}\left(v^{N}\right)=\lim _{N \rightarrow \infty} f^{*}\left(v^{N}\right)=f^{\#}\left(v_{0}\right) .
$$

Thus $g(v) \leqslant f^{\#}(v)$ and, consequently, $f^{(q c)}(v) \leqslant f^{\#}(v)$ for all $v \in \mathbb{R}^{n m}$. On the other hand, by Theorem 3.16(1) and Theorem 3.19, $f^{\#}$ is admissible in the formation of $f^{(q c)}$, and we conclude that $f^{\#}(v) \leqslant f^{(q c)}(v)$ and $f^{\#}(v)=f^{(q c)}(v)$ for all $v \in \mathbb{R}^{n m}$.

Proof of Theorem 4.2. (1) follows from Theorem 3.19. and Theorem 4.1.

(2) The inequalities result from Theorem 2.18(1), Part (1) and Theorem 2.7.

Proof of Theorem 4.3. The identities follow from Theorem 4.2(2) and Theorem 2.6.

\subsection{An example of a function $f \in \mathcal{F}_{\mathrm{K}}$ with $f^{(q c)} \neq f^{*}$}

Let $\mathrm{K}$ be the four-dimensional cube $\mathrm{K}=[-1,1]^{4} \subset \mathbb{R}^{2 \times 2}$. We provide an example of a function $f \in \mathcal{F}_{\mathrm{K}}$ where $f^{(q c)}$ and $f^{*}$ disagree. Furthermore, $f^{*}$ is quasiconvex but not rank one convex. Thereby we make use of the notations from [41], pp. 242-244, and the calculation of $f^{*}$ ibid., p. 246, Theorem 7. 
Definition 4.4. Given the four-dimensional cube $\mathrm{K}=[-1,1]^{4} \subset \mathbb{R}^{2 \times 2}$. Consider the function $f: \mathbb{R}^{2 \times 2} \rightarrow \overline{\mathbb{R}}$, $f \in \mathcal{F}_{\mathrm{K}}$, defined by

$$
f(v)=\left\{\begin{array}{c|l}
a+b+c+\left(1-d^{2}\right) & \mid v \in \mathrm{K} ; \\
+\infty & v \in \mathbb{R}^{2 \times 2} \backslash \mathrm{K} .
\end{array}\right.
$$

Theorem 4.5 (Properties of $f^{*}$ and $f^{(q c)}$ for the function $f$ from Def. 4.4).

(1) (Calculation of $f^{*}$ and $\left.f^{(q c)}\right)^{52}$ Using the notations from [41], pp. $242-244$, the envelopes of $f$ take the following shape:

$$
\begin{aligned}
f^{*}(v) & = \begin{cases}a+b+c+\left(1-d^{2}\right) & \mid v \in \operatorname{ext}(\mathrm{K}) \text { or } v \in \operatorname{ri}(\Phi), \Phi \in \mathcal{G}_{1} \cup \mathcal{G}_{2,3} \cup \mathcal{G}_{3,4} ; \\
a+b+c & \mid v \in \operatorname{int}(\mathrm{K}) \text { or } v \in \operatorname{ri}(\Phi), \Phi \in \mathcal{G}_{2,1} \cup \mathcal{G}_{2,2} \cup \mathcal{G}_{3,1} \cup \mathcal{G}_{3,2} \cup \mathcal{G}_{3,3} ; \\
+\infty & \mid v \in \mathbb{R}^{2 \times 2} \backslash \mathrm{K}\end{cases} \\
f^{(q c)}(v) & = \begin{cases}a+b+c & \mid v \in \mathrm{K} ; \\
+\infty & \mid v \in \mathbb{R}^{2 \times 2} \backslash \mathrm{K} .\end{cases}
\end{aligned}
$$

(2) $\left(f^{(q c)}\right.$ and $f^{*}$ do not agree) There is $f^{(q c)}(v)<f^{*}(v)$ precisely for all points $v \in \operatorname{ri}(\Phi), \Phi \in\left\{\mathrm{S}_{1}, \mathrm{~S}_{2}, \mathrm{~S}_{5}, \mathrm{~S}_{6}\right.$, $\left.\mathrm{S}_{13}, \mathrm{~S}_{14}, \mathrm{~S}_{17}, \mathrm{~S}_{18}\right\} \cup\left\{\mathrm{Q}_{3}, \mathrm{Q}_{4}, \mathrm{Q}_{9}, \mathrm{Q}_{10}, \mathrm{Q}_{13}, \mathrm{Q}_{14}, \mathrm{Q}_{17}, \mathrm{Q}_{18}\right\} \cup\left\{\mathrm{W}_{5}, \mathrm{~W}_{6}\right\}$.

(3) (Generalized convexity properties of $f^{*}$ ) The function $f^{*}$ is quasiconvex but not rank one convex.

Proof of Theorem 4.5. (1) The calculation of $f^{*}(v)$ on the faces of K has been carried out in [41], p. 247 f., so that we only have to determine $f^{(q c)}$. By Definition 3.11 and Theorem 4.1, $f^{*}$ and $f^{(q c)}$ may differ at most on $\partial \mathrm{K}$, and in its points, we get $f^{(q c)}\left(v_{0}\right)$ by limit passage $\lim _{v \rightarrow v_{0}} f^{*}(v)$ along the rays starting from $\mathfrak{o}$. In our case, $f^{(q c)} \mid \mathrm{K}$ arises as the continuous extension of $f^{*} \mid \operatorname{int}(\mathrm{K})$ to the boundary.

(2) Precisely for the points $v$ from the relative interior of the faces specified in the assertion, it holds that $f^{*}(v)-f^{(q c)}(v)=\left(1-d^{2}\right)$ and $|d|<1$.

(3) By Theorem 3.8(1), $f^{*} \mid \mathrm{K}$ is upper semicontinuous, and the conditions (1) and (2) from Definition 2.9 are satisfied. By Theorem 3.17(2), Morrey's integral inequality holds for all $v_{0} \in \operatorname{int}(\mathrm{K})$, and by Theorem 2.11(1), the same is true for all $v_{0} \in \mathbb{R}^{2 \times 2} \backslash \mathrm{K}$. Now we use Theorem $2.11(3)$ to examine its validity on the faces of $\mathrm{K}$ and distinguish the following cases:

(i) For $v_{0} \in \operatorname{ext}(\mathrm{K})$ as well as $v_{0} \in \operatorname{ri}(\Phi), \Phi \in \mathcal{G}_{1} \cup \mathcal{G}_{2,3}$, precisely the function $x=\mathfrak{o}$ is feasible when forming the infimum in (2.12) in Theorem 2.11(3) (see [41], p. 244, Proof of Thm. 6, (ii)).

(ii) For $v_{0} \in \operatorname{ri}(\Phi)$ with $\Phi \in \mathcal{G}_{2,1} \cup \mathcal{G}_{2,2} \cup \mathcal{G}_{3,1} \cup \mathcal{G}_{3,2} \cup \mathcal{G}_{3,3}$, Morrey's integral inequality takes by Part (1) the following shape:

$$
\begin{array}{r}
f^{*}\left(v_{0}\right)=\inf \left\{\frac{1}{|\Omega|} \int_{\Omega}\left(a_{0}+\frac{\partial x_{1}}{\partial t_{1}}(t)+b_{0}+\frac{\partial x_{1}}{\partial t_{2}}(t)+c_{0}+\frac{\partial x_{2}}{\partial t_{1}}(t)\right) \mathrm{d} t_{1} \mathrm{~d} t_{2} \mid\right. \\
\left.x \in W_{0}^{1, \infty}\left(\Omega, \mathbb{R}^{2}\right), v+J x(t) \in \Phi(\forall) t \in \Omega\right\} .
\end{array}
$$

By Lemma 5.1(1), we get on the right-hand side as well

$$
a_{0}+b_{0}+c_{0}=f^{*}\left(v_{0}\right),
$$

and the inequality is satisfied.

\footnotetext{
${ }^{52}$ Calculation of $f^{*}:[41]$, p. 246 , Theorem 7 .
} 
(iii) For $v_{0} \in \operatorname{ri}(\Phi)$ with $\Phi \in\left\{\mathrm{W}_{5}, \mathrm{~W}_{6}\right\}$, Morrey's integral inequality reads as follows:

$$
\begin{array}{r}
f^{*}\left(v_{0}\right)=\inf \left\{\frac{1}{|\Omega|} \int_{\Omega}\left(a_{0}+\frac{\partial x_{1}}{\partial t_{1}}(t)+b_{0}+\frac{\partial x_{1}}{\partial t_{2}}(t)+c_{0}+\frac{\partial x_{2}}{\partial t_{1}}(t)+\left(1-\left(d_{0}+\frac{\partial x_{2}}{\partial t_{2}}(t)\right)^{2}\right)\right) \mathrm{d} t_{1} \mathrm{~d} t_{2} \mid\right. \\
\left.x \in W_{0}^{1, \infty}\left(\Omega, \mathbb{R}^{2}\right), v+J x(t) \in \Phi(\forall) t \in \Omega\right\} .
\end{array}
$$

In analogy to [41], p. 246, Proof of Theorem 6, (iv), only functions with $x_{2}=\mathfrak{o}$ are feasible in the forming of the infimum. It follows again from Lemma 5.1(1) that the right-hand side takes the value

$$
a_{0}+b_{0}+c_{0}+\left(1-\left(d_{0}\right)^{2}\right)=f^{*}\left(v_{0}\right)
$$

and the inequality is satisfied in this case as well.

Summing up, we see that $f^{*}$ is quasiconvex. However, the function fails to be rank one convex. For example, along the edge $\mathrm{S}_{1} \in \mathfrak{F}_{1}, f^{*}(v)=f(v)=a+b+c+\left(1-d^{2}\right)$ is concave instead of convex (Part (1)) but $\left.\left.\mathrm{S}_{1}=\left\{\lambda \cdot\left(\begin{array}{cc}1 & 1 \\ 1 & -1\end{array}\right)\right\}+(1-\lambda) \cdot\left(\begin{array}{ll}1 & 1 \\ 1 & 1\end{array}\right)\right\} \mid 0 \leqslant \lambda \leqslant 1\right\}$ is a rank one direction.

\subsection{An example of envelopes $f^{*}$ and $f^{(q c)}$ changing discontinuously with the Hausdorff approximation of $\mathbf{K}$}

In our last example we ask to what extent the forming of the envelopes $f^{*}$ and $f^{(q c)}$ is compatible with the Hausdorff approximation of $\mathrm{K}^{53}$. To see this, we approximate the four-dimensional cube $\mathrm{K}$ by a sequence of "smooth" convex bodies $\left\{\mathrm{K}^{N}\right\}$ (Thm. 5.5(2)) and $f$ from Definition 4.4. by a sequence of functions $f^{N} \in \mathcal{F}_{\mathrm{K}^{N}}$. It turns out that on $\mathrm{K}$, neither $f^{*}$ nor $f^{(q c)}$ will be approximated in pointwise convergence by $\left\{\left(f^{N}\right)^{*}\right\}$ resp. $\left\{\left(f^{N}\right)^{(q c)}\right\}$.

Definition 4.6. Again, we consider the four-dimensional cube $K=[-1,1]^{4} \subset \mathbb{R}^{2 \times 2}$ and the function $f: \mathbb{R}^{2 \times 2} \rightarrow \overline{\mathbb{R}}$ with

$$
f(v)=\left\{\begin{array}{cl}
a+b+c+\left(1-d^{2}\right) & \mid v \in \mathrm{K} \\
+\infty & \mid v \in \mathbb{R}^{2 \times 2} \backslash \mathrm{K}
\end{array}\right.
$$

from Definition 4.4. Let a sequence of convex bodies $\mathrm{K}^{N} \subset \mathbb{R}^{2 \times 2}$ with the properties (a) $\mathrm{K}^{N+1} \subset \mathrm{K}^{N}$, (b) $\mathrm{K} \subset \mathrm{K}^{N} \subset\left(\mathrm{K}+\mathrm{K}\left(\mathfrak{o}, \frac{1}{N}\right)\right)$ and (c) $\partial \mathrm{K}^{N}=\operatorname{ext}\left(\mathrm{K}^{N}\right)$ for all $\mathrm{N} \in \mathbb{N}$ be given. Further, we consider the functions $f^{N}: \mathbb{R}^{2 \times 2} \rightarrow \overline{\mathbb{R}}$ defined by

$$
f^{N}(v)=\left\{\begin{array}{c|c}
a+b+c+\left(1-d^{2}\right) \mid v \in \mathrm{K}^{N} \\
+\infty & \mid v \in \mathbb{R}^{2 \times 2} \backslash \mathrm{K}^{N} .
\end{array}\right.
$$

Theorem 4.7 (Approximation of $\mathrm{K}, f, f^{*}$ and $f^{(q c)}$ ).

(1) The sequence $\left\{\mathrm{K}^{N}\right\}$ converges to $\mathrm{K}$ in Hausdorff distance.

(2) For all $v \in \mathrm{K}$ and all sequences of points $v^{N} \in \mathrm{K}^{N}$ with $v^{N} \rightarrow v$ it holds that $\lim _{N \rightarrow \infty} f^{N}\left(v^{N}\right)=f(v)$. In particular, $\left\{f^{N}\right\}$ approximates $f$ in everywhere pointwise convergence.

(3) There exists a point $v_{0} \in \partial \mathrm{K}$ such that

$$
\lim _{N \rightarrow \infty}\left(f^{N}\right)^{*}\left(v^{N}\right) \neq f^{*}\left(v_{0}\right) \text { and } \lim _{N \rightarrow \infty}\left(f^{N}\right)^{(q c)}\left(v^{N}\right) \neq f^{(q c)}\left(v_{0}\right)
$$

holds for every sequence of points $v^{N} \in \partial \mathrm{K}^{N}$ with $v^{N} \rightarrow v_{0}$.

\footnotetext{
${ }^{53} C f$. [25], p. 356, Lemma 7.3 .
} 
Proof of Theorem 4.7.

(1) is clear from the definition of the Hausdorff distance.

(2) The function $\tilde{f}: \mathbb{R}^{2 \times 2} \rightarrow \mathbb{R}$ defined by

$$
\widetilde{f}(v)=a+b+c+\left(1-d^{2}\right)
$$

is continuous. Consequently, we have for all $v \in \mathrm{K}$ :

$$
\lim _{N \rightarrow \infty} f^{N}\left(v^{N}\right)=\lim _{N \rightarrow \infty} \widetilde{f}\left(v^{N}\right)=\widetilde{f}(v)=f(v) .
$$

This is true, in particular, if $v^{N}=v \in \mathrm{K}$ for all $N \in \mathbb{N}$. For $v \notin \mathrm{K}$, there is Dist $(v, \mathrm{~K})>0$ (since $\mathrm{K}$ is compact), and for all sufficiently large indices $N$ it holds that

$$
f^{N}(v)=f(v)=+\infty
$$

(3) Take the point $v_{0}=\left(\begin{array}{ll}1 & 1 \\ 0 & 0\end{array}\right) \in \operatorname{ri}\left(\mathrm{Q}_{1}\right) \subset \mathrm{K}$ and consider a sequence of points $v^{N} \in \partial \mathrm{K}^{N}$ with $v^{N} \rightarrow v_{0}$. Then $v^{N}$ can be represented as

$$
v^{N}=\left(\begin{array}{ll}
1 & 1 \\
0 & 0
\end{array}\right)+\left(\begin{array}{ll}
a^{N} & b^{N} \\
c^{N} & d^{N}
\end{array}\right) \quad \text { with } \lim _{N \rightarrow \infty}\left(\left(a^{N}\right)^{2}+\left(b^{N}\right)^{2}+\left(c^{N}\right)^{2}+\left(d^{N}\right)^{2}\right)=0 .
$$

Since $\partial \mathrm{K}^{N}=\operatorname{ext}\left(\mathrm{K}^{N}\right)$, we find with Theorem 3.4(3):

$$
\left(f^{N}\right)^{*}\left(v^{N}\right)=f^{N}\left(v^{N}\right)=2+a^{N}+b^{N}+c^{N}+\left(1-\left(d^{N}\right)^{2}\right) \quad \Longrightarrow \quad \lim _{N \rightarrow \infty}\left(f^{N}\right)^{*}\left(v^{N}\right)=3 .
$$

On the other hand, since $\mathrm{Q}_{1} \in \mathfrak{F}_{2,1}$ it follows from Theorem 4.5(1): $f^{*}\left(v_{0}\right)=2$. By Theorem 2.18(1), from $\partial \mathrm{K}^{N}=\operatorname{ext}\left(\mathrm{K}^{N}\right)$ it follows similarly that

$$
\left(f^{N}\right)^{(q c)}\left(v^{N}\right)=f^{N}\left(v^{N}\right)=2+a^{N}+b^{N}+c^{N}+\left(1-\left(d^{N}\right)^{2}\right) \Longrightarrow \lim _{N \rightarrow \infty}\left(f^{N}\right)^{(q c)}\left(v^{N}\right)=3
$$

while Theorem 4.5(1) yields $f^{(q c)}\left(v_{0}\right)=2$.

\section{Appendix}

\subsection{Two properties of Jacobi matrices}

Lemma 5.1. Given a set $\Omega \subset \mathbb{R}^{m}$ as the closure of a strongly Lipschitz domain and a function $x \in$ $W_{0}^{1, \infty}\left(\Omega, \mathbb{R}^{n}\right)$.

(1) Then for all $1 \leqslant i \leqslant n, 1 \leqslant j \leqslant m$ it holds that $\int_{\Omega} \frac{\partial x_{i}}{\partial t_{j}}(t) \mathrm{d} t=0$.

(2) For any hyperplane $\mathrm{H}=\left\{v \in \mathbb{R}^{n m} \mid\langle a, v\rangle=0\right\}$ with $a \neq \mathfrak{o}$, we have the implication: $\langle a, J x(t)\rangle \geqslant 0$

$(\forall) t \in \Omega \Longrightarrow\langle a, J x(t)\rangle=0(\forall) t \in \Omega$.

Proof of Lemma 5.1 .

(1) Application of Gauss' theorem (see [22], p. 133, Thm. 1, (ii)) to $f(t)=x_{i}(t)$ as well as $\varphi_{1}(t), \ldots, \varphi_{j-1}(t)$, $\varphi_{j+1}(t), \ldots, \varphi_{m}(t) \equiv 0$ and $\varphi_{j}(t) \equiv 1$ leads to

$$
\int_{\Omega} x_{i}(t) \operatorname{div} \varphi(t) \mathrm{d} t+\int_{\Omega} \sum_{k=1}^{m} \frac{\partial x_{i}}{\partial t_{k}}(t) \varphi_{k}(t) \mathrm{d} t=\int_{\partial \Omega} \sum_{k=1}^{m} \mathfrak{n}_{k}(s) \varphi_{k}(s) x_{i}(s) \mathrm{d} s .
$$

Since $x_{i} \mid \partial \Omega \equiv 0$ and $\operatorname{div} \varphi \equiv 0$ the claim follows. 
(2) Assume to the contrary that a set $\Omega_{1} \subseteq \Omega$ with $\left|\Omega_{1}\right|>0$ and $\langle a, J x(t)\rangle>0(\forall) t \in \Omega_{1}$ exists. Then it follows from Part (1):

$$
\int_{\Omega} \sum_{i, j} a_{i j} \frac{\partial x_{i}}{\partial t_{j}}(t) \mathrm{d} t=\int_{\Omega_{1}} \sum_{i, j} a_{i j} \frac{\partial x_{i}}{\partial t_{j}}(t) \mathrm{d} t+\int_{\Omega \backslash \Omega_{1}} \sum_{i, j} a_{i j} \frac{\partial x_{i}}{\partial t_{j}}(t) \mathrm{d} t=S_{1}+S_{2}=0
$$

and we arrive at a contradiction since $S_{1}>0$ and $S_{2} \geqslant 0$.

\subsection{Convex bodies}

Lemma 5.2 (Decomposition of $\mathrm{K}$ into faces) ${ }^{54}$. For any convex body $\mathrm{K} \subset \mathbb{R}^{n m}$, the family $\{\operatorname{ri}(\Phi) \mid \Phi \neq \varnothing$ is a face of $\mathrm{K}\}$ forms a decomposition of $\mathrm{K}$ into mutually disjoint subsets.

Lemma 5.3 (Measure-theoretical properties of $\mathrm{K}$ and $\partial \mathrm{K})^{55}$. Any convex body $\mathrm{K} \subset \mathbb{R}^{n m}$ is a nmdimensional Borel set, and its boundary $\partial \mathrm{K}$ is a $\mathrm{nm}$-dimensional Lebesgue null set (thus $\mathrm{K}$ is even squarable).

Lemma 5.4 (Measure-theoretical properties of ext $(\mathrm{K}))^{56}$. If $\mathrm{K} \subset \mathbb{R}^{n m}$ is a convex body then ext $(\mathrm{K}) \subset$ $\mathbb{R}^{n m}$ is a $G_{\delta}$-set, and its characteristic function $\mathbb{1}_{\text {ext }(\mathrm{K})}$ belongs to the second Baire class.

Theorem 5.5 (Approximation of convex bodies in Hausdorff distance). Given a convex body $\mathrm{K} \subset \mathbb{R}^{n m}$ and a number $\varepsilon>0$.

(1) (Approximation by polytopes) Then there exist polytopes $\mathrm{P}, \mathrm{Q} \subset \mathbb{R}^{n m}$ with $\operatorname{Dim}(\mathrm{P})=\operatorname{Dim}(\mathrm{Q})=n m$ and $\mathrm{P} \subseteq \mathrm{K} \subseteq \mathrm{Q} \subseteq(\mathrm{P}+\mathrm{K}(\mathfrak{o}, \varepsilon))$.

(2) (Approximation by "smooth" bodies) Then there exist convex bodies $\mathrm{P}, \mathrm{Q} \subset \mathbb{R}^{\text {nm }}$ with $\operatorname{Dim}(\mathrm{P})=$ $\operatorname{Dim}(\mathrm{Q})=n m$ and the following properties: All boundary points of $\mathrm{P}$ resp. $\mathrm{Q}$ are at the same time extremal points; through every boundary point of $\mathrm{P}$ resp. Q passes precisely one supporting hyperplane, and it holds $\mathrm{P} \subseteq \mathrm{K} \subseteq \mathrm{Q} \subseteq(\mathrm{P}+\mathrm{K}(\mathfrak{o}, \varepsilon))$

Proof of Theorem 5.5. (1) ${ }^{57}$ The compact set $\mathrm{K}$ can be covered by finitely many open cubes $\mathrm{Q}\left(v_{s}, \delta\right)$ in such a way that $v_{s} \in \mathrm{K}, \mathrm{Q}\left(v_{s}, \delta\right) \subset \mathrm{K}\left(v_{s}, \varepsilon\right)$ for $1 \leqslant s \leqslant r$ and $\mathrm{K} \subseteq \bigcup_{s=1}^{r} \mathrm{Q}\left(v_{s}, \delta\right)$ hold. We may further assume that $(n m+1)$ of the centers $v_{s}$ are affinely independent. Then $\mathrm{P}=\operatorname{co}\left(\left\{v_{1}, \ldots, v_{r}\right\}\right)$ and $\mathrm{Q}=$ co $\left(\bigcup_{s=1}^{r} \operatorname{cl}\left(\mathrm{Q}\left(v_{s}, \delta\right)\right)\right)$ admit the claimed properties: obviously $\mathrm{P} \subseteq \mathrm{K} \subseteq \mathrm{Q}$ and $\mathrm{P} \subseteq \mathrm{K} \subseteq(\mathrm{P}+\mathrm{K}(\mathfrak{o}, \varepsilon))$ hold as well as $\mathrm{Q}=\mathrm{co}\left(\left\{v_{1}, \ldots, v_{r}\right\}+\mathrm{Q}(\mathfrak{o}, \delta)\right)=\mathrm{P}+\mathrm{Q}(\mathfrak{o}, \delta) \subset \mathrm{P}+\mathrm{K}(\mathfrak{o}, \varepsilon)$.

(2) By [35], p. 40, Corollary 1.7.3, the support function $h_{\mathrm{Q}}$ of a convex body is differentiable in a point $v \neq \mathfrak{o}$ iff the intersection of $\mathrm{Q}$ with the supporting hyperplane $\mathrm{H}_{v}=\left\{w \in \mathbb{R}^{n m} \mid\langle v, w\rangle=h_{\mathrm{Q}}(v)\right\}$ is a singleton. Then $v$ belongs to ext (Q). [35], p. 158, Theorem 3.3.1, guarantees the existence of convex bodies which admit support functions within $C^{\infty}\left(\mathbb{R}^{n m} \backslash\{\mathfrak{o}\}, \mathbb{R}\right)$ and approximate $\mathrm{K}$ in Hausdorff distance. Following the remark ibid., p. 160, we may add norm balls to these bodies, thus arriving at the assertion.

\subsection{The convex envelope}

Theorem 5.6 (Representation of the convex envelope $\left.f^{c}\right)^{58}$. Given a nonempty, closed, convex set $\mathrm{C} \subseteq \mathbb{R}^{n m}$ and a function $f: \mathrm{C} \rightarrow \overline{\mathbb{R}}$ bounded from below, then $f^{c}$ admits the representation

$$
f^{c}(v)=\inf \left\{\sum_{s=1}^{n m+1} \lambda_{s} f\left(v_{s}\right) \mid \sum_{s} \lambda_{s}=1, \sum_{s} \lambda_{s} v_{s}=v, 0 \leqslant \lambda_{s} \leqslant 1, v_{s} \in \mathrm{C}, 1 \leqslant s \leqslant n m+1\right\} .
$$

\footnotetext{
$54[5]$, p. 32, Corollary 5.7, resp. [35], p. 62, Theorem 2.1.2.

$55[21]$, p. 68 , Theorem 7.7. and Corollary 7.8.

$56[35]$, p. 66 .

${ }^{57}$ Cf. [35], p. 54 f., Theorem 1.8.13.

58 [33], p. 157, Corollary 17.1.5; the theorem is still valid with the restriction to C.
} 
Theorem 5.7 (Properties of $f^{c}$ for $f \in \mathcal{F}_{\mathrm{K}}$ ). For any function $f \in \mathcal{F}_{\mathrm{K}}$, the following assertions hold: $(1)^{59} f^{c}$ is lower semicontinuous.

(2) Within the definition of $f^{c}$, it suffices to form the supremum over convex functions with finite values resp. over affine functions only:

$$
\begin{aligned}
f^{c}(v) & =\sup \left\{g(v) \mid g: \mathbb{R}^{n m} \rightarrow \mathbb{R} \text { convex, } g(v) \leqslant f(v) \forall v \in \mathbb{R}^{n m}\right\} \\
& =\sup \left\{g(v) \mid g: \mathbb{R}^{n m} \rightarrow \mathbb{R} \quad \text { affine, } g(v) \leqslant f(v) \forall v \in \mathbb{R}^{n m}\right\} .
\end{aligned}
$$

(3) For all $v \in \mathbb{R}^{n m} \backslash \mathrm{K}$, it holds that $f^{c}(v)=+\infty$.

(4) If $\Phi$ is a $k$-dimensional face of $\mathrm{K}, 0 \leqslant k \leqslant n m$, then $f^{c}(v)<+\infty$ for all $v \in \Phi$, and we have

$$
f^{c}(v)=\inf \left\{\sum_{s=1}^{k+1} \lambda_{s} f\left(v_{s}\right) \mid \sum_{s} \lambda_{s}=1, \sum_{s} \lambda_{s} v_{s}=v, 0 \leqslant \lambda_{s} \leqslant 1, v_{s} \in \Phi, 1 \leqslant s \leqslant k+1\right\} .
$$

(5) In particular, $f^{c}(v)=f(v)$ for all $v \in \operatorname{ext}(\mathrm{K})$.

(6) If $\Phi$ is a $k$-dimensional face of $\mathrm{K}, 0 \leqslant k \leqslant n m$, then the restriction $f^{c} \mid \Phi$ is lower semicontinuous, and the restriction $f^{c} \mid \operatorname{ri}(\Phi)$ is continuous.

Proof of Theorem 5.7(6). The restriction of the lower semicontinuous function $f^{c}$ to the face $\Phi$ is lower semicontinuous as well since $\Phi$ consists of accumulation points only (see [7], p. 136, Thm. 1). The second assertion follows from [33], p. 82, Theorem 10.1.

\section{REFERENCES}

[1] J.A. Andrejewa and R. Klötzler, Zur analytischen Lösung geometrischer Optimierungsaufgaben mittels Dualität bei Steuerungsproblemen. Teil I. Z. Angew. Math. Mech. 64 (1984) 35-44.

[2] J.A. Andrejewa and R. Klötzler, Zur analytischen Lösung geometrischer Optimierungsaufgaben mittels Dualität bei Steuerungsproblemen. Teil II. Z. Angew. Math. Mech. 64 (1984) 147-153.

[3] G. Aubert and P. Kornprobst, Mathematical Problems in Image Processing: Partial Differential Equations and the Calculus of Variations. 2nd Edn., Springer, New York etc. (2006).

[4] J.M. Ball and F. Murat, $W^{1, p}$-quasiconvexity and variational problems for multiple integrals. J. Funct. Anal. 58 (1984) 225-253.

[5] A. Brøndsted, An Introduction to Convex Polytopes. Springer, New York - Heidelberg - Berlin (1983).

[6] C. Brune, H. Maurer and M. Wagner, Edge detection within optical flow via multidimensional control. BTU Cottbus, PreprintReihe Mathematik, Preprint Nr. M-02/2008 (submitted).

[7] C. Carathéodory, Vorlesungen über reelle Funktionen. 3rd Edn., Chelsea, New York (1968).

[8] E. Casadio Tarabusi, An algebraic characterization of quasi-convex functions. Ricerche di Mat. 42 (1993) 11-24.

[9] F.H. Clarke, Optimization and Nonsmooth Analysis. 2nd Edn., SIAM, Philadelphia (1990).

[10] L. Collatz and W. Wetterling, Optimierungsaufgaben, 2nd Edn., Heidelberger Taschenbücher 15. Springer, Berlin - Heidelberg New York (1971).

[11] B. Dacorogna, Quasiconvexity and relaxation of nonconvex problems in the calculus of variations. J. Funct. Anal. 46 (1982) $102-118$.

[12] B. Dacorogna, Direct Methods in the Calculus of Variations. 2nd Edn., Springer, New York etc. (2008).

[13] B. Dacorogna and N. Fusco, Semi-continuité des fonctionnelles avec contraintes du type "det $\nabla u>0$ ". Boll. Un. Mat. Ital. B (6) 4 (1985) 179-189.

[14] B. Dacorogna and P. Marcellini, General existence theorems for Hamilton-Jacobi equations in the scalar and vectorial case. Acta Math. 178 (1997) 1-37.

[15] B. Dacorogna and P. Marcellini, Cauchy-Dirichlet problem for first order nonlinear systems. J. Funct. Anal. 152 (1998) 404-446.

[16] B. Dacorogna and P. Marcellini, Implicit Partial Differential Equations. Birkhäuser, Boston - Basel - Berlin (1999).

[17] B. Dacorogna and A.M. Ribeiro, On some definitions and properties of generalized convex sets arising in the calculus of variations, in Recent Advances on Elliptic and Parabolic Issues, M. Chipot and H. Ninomiya Eds., Proceedings of the 2004 Swiss-Japanese Seminar: Zurich, Switzerland, 6-10 December 2004, World Scientific, Singapore (2006) 103-128.

\footnotetext{
${ }^{59}$ Parts (1)-(5): $c f .[42]$, p. 128 f., Theorem 10.12.
} 
[18] R. De Arcangelis and E. Zappale, The relaxation of some classes of variational integrals with pointwise continuous-type gradient constraints. Appl. Math. Optim. 51 (2005) 251-257.

[19] R. De Arcangelis, S. Monsurrò and E. Zappale, On the relaxation and the Lavrentieff phenomenon for variational integrals with pointwise measurable gradient constraints. Calc. Var. Partial Differential Equations 21 (2004) $357-400$.

[20] I. Ekeland and R. Témam, Convex Analysis and Variational Problems. 2nd Edn., SIAM, Philadelphia (1999).

[21] J. Elstrodt, Maß- und Integrationstheorie. Springer, New York - Heidelberg - Berlin (1996).

[22] L.C. Evans and R.F. Gariepy, Measure Theory and Fine Properties of Functions. CRC Press, Boca Raton etc. (1992).

[23] A.D. Ioffe and V.M. Tichomirow, Theorie der Extremalaufgaben. VEB Deutscher Verlag der Wissenschaften, Berlin (1979).

[24] B. Kawohl, From Mumford-Shah to Perona-Malik in image processing. Math. Meth. Appl. Sci. 27 (2004) $1803-1814$.

[25] D. Kinderlehrer and P. Pedregal, Characterizations of Young measures generated by gradients. Arch. Rat. Mech. Anal. 115 (1991) 329-365.

[26] J. Kristensen, On the non-locality of quasiconvexity. Ann. Inst. H. Poincaré Anal. Non Linéaire 16 (1999) 1-13.

[27] J.B. Kruskal, Two convex counterexamples: A discontinuous envelope function and a nondifferentiable nearest-point mapping. Proc. Amer. Math. Soc. 23 (1969) 697-703.

[28] M. Kružík, Bauer's maximum principle and hulls of sets. Calc. Var. Partial Differential Equations 11 (2000) $321-332$.

[29] M. Kružík, Quasiconvex extreme points of convex sets, in Elliptic and Parabolic Problems, J. Bemelmans, B. Brighi, A. Brillard, M. Chipot, F. Conrad, I. Shafrir, V. Valente and G. Vergara-Caffarelli Eds., World Scientific Publishing, River Edge (2002) $145-151$.

[30] K.A. Lur'e (К.А. Лурье), Оптимальное управление в задачах математической физики. Наука, Москва (1975).

[31] C.B. Morrey, Quasi-convexity and the lower semicontinuity of multiple integrals. Pacific J. Math. 2 (1952) 25-53.

[32] S. Pickenhain and M. Wagner, Piecewise continuous controls in Dieudonné-Rashevsky type problems. J. Optim. Theory Appl. 127 (2005) 145-163.

[33] R.T. Rockafellar, Convex Analysis. 2nd Edn., Princeton University Press, Princeton (1972).

[34] R.T. Rockafellar and R.J.-B. Wets, Variational Analysis, Grundlehren 317. Springer, Berlin etc. (1998).

[35] R. Schneider, Convex Bodies: The Brunn-Minkowski Theory. Cambridge University Press, Cambridge (1993).

[36] K. Schulz and B. Schwartz, Finite extensions of convex functions. Math. Operationsforschung Statist. Ser. Optimization 10 (1979) 501-509.

[37] V. Šverák, Rank-one convexity does not imply quasiconvexity. Proc. Roy. Soc. Edinburgh Ser. A 120 (1992) $185-189$.

[38] T.W. Ting, Elastic-plastic torsion of convex cylindrical bars. J. Math. Mech. 19 (1969) 531-551.

[39] T.W. Ting, Elastic-plastic torsion problem III. Arch. Rat. Mech. Anal. 34 (1969) 228-244.

[40] M. Wagner, Erweiterungen des mehrdimensionalen Pontrjaginschen Maximumprinzips auf meßbare und beschränkte sowie distributionelle Steuerungen. Ph.D. thesis, Universität Leipzig, Germany (1996).

[41] M. Wagner, Nonconvex relaxation properties of multidimensional control problems, in Recent Advances in Optimization, A. Seeger Ed., Lecture Notes in Economics and Mathematical Systems 563, Springer, Berlin etc. (2006) 233-250.

[42] M. Wagner, Mehrdimensionale Steuerungsprobleme mit quasikonvexen Integranden. Habilitation thesis, Brandenburgische Technische Universität Cottbus, Cottbus, Germany (2006).

[43] M. Wagner, Pontryagin's maximum principle for multidimensional control problems in image processing. J. Optim. Theory Appl. (to appear).

[44] K. Zhang, On the structure of quasiconvex hulls. Ann. Inst. H. Poincaré Anal. Non Linéaire 15 (1998) 663-686.

[45] K. Zhang, On the quasiconvex exposed points. ESAIM: COCV 6 (2001) 1-19 (electronic). 